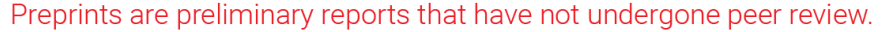 They should not be considered conclusive, used to inform clinical practice, or referenced by the media as validated information. \\ Similarity measures of Pythagorean fuzzy soft sets and clustering analysis
}

\section{Athira T M}

National Institute of Technology Calicut

Sunil Jacob John ( $\nabla$ sunil@nitc.ac.in )

National Institute of Technology Calicut https://orcid.org/0000-0002-6333-2884

\section{Harish Garg}

Thapar University

\section{Research Article}

Keywords: Pythagorean fuzzy soft sets, Similarity measures, Clustering algorithms

Posted Date: July 31st, 2021

DOl: https://doi.org/10.21203/rs.3.rs-226310/v1

License: (1) This work is licensed under a Creative Commons Attribution 4.0 International License.

Read Full License 


\title{
Similarity measures of Pythagorean fuzzy soft sets and clustering analysis
}

\author{
Athira T M • Sunil Jacob John · Harish \\ Garg
}

Received: date / Accepted: date

\begin{abstract}
Pythagorean fuzzy set (PFS) is a broadening of intuitionistic fuzzy set that can represent the situations where the sum of membership and the non-membership values exceeds one. Adding parameterization to PFS we obtain a structure named as Pythagorean fuzzy soft set (PFSS). It has a higher capacity to deal with vagueness as it captures both the structures of a PFS and a soft set. Several practical situations demand the measure of similarity between two structures, whose sum of membership value and non-membership value exceeds one. There are no existing tools to measure the similarity between PFSS and this paper put forward similarity measures for PFSS. An axiomatic definition for similarity measure is proposed for PFSS and certain expressions for similarity measure are introduced. Further, some theorems which express the properties of similarity measures are proved. A comparative study between proposed expressions for similarity measure is carried out. Also, a clustering algorithm based on PFSS is introduced by utilizing the proposed similarity measure.
\end{abstract}

keywords: Pythagorean fuzzy soft sets, Similarity measures, Clustering algorithms

Athira T M

Department of Mathematics,

National Institute of Technology Calicut-673 601, Kerala, INDIA

E-mail: athiratm999maths@gmail.com

Sunil Jacob John

Department of Mathematics,

National Institute of Technology Calicut-673 601, Kerala, INDIA

E-mail: sunil@nitc.ac.in

Harish Garg

School of Mathematics,

Thapar lnstitute of Engineering and Technology

Patiala-147004, Panjab, INDIA

E-mail: harishg58iitr@gmail.com 


\section{Introduction}

A measure of analogy between the objects is necessary for many real-life problems like clustering, pattern recognition, sequence alignment, medical diagnosis, etc. This motivates to introduce similarity measures and caste its useful expressions. Since all the problems mentioned above have to cope with uncertainties, the notion of similarity measure is explored in many generalized set-theoretical concepts that can handle vagueness.

In 1965, Zadeh's fuzzy set (FS) theory [35] was a paradigm shift in this regard, as it decreases the space between real life and mathematics. The FS points out the membership value of objects in $[0,1]$ and which surpasses the crisp set (whose range of membership value is 0 and 1 ). For this reason, there are a lot of research works on FSs in the literature and many extensions are attained. We mention some of them here. Intuitionistic fuzzy set (IFS), introduced by Atanassov [2], is capable to explain the objects' membership value together with nonmembership value, $\mu$ and $\nu$ respectively with the property, $0 \leq \mu+\nu \leq 1$. Torra figured out the Hesitant fuzzy set (HFS) [30], which can overcome the difficulty that hesitancy of giving membership value by establishing more than one membership value of an object. Yager's Pythagorean fuzzy set (PFS) [34] is a broadening of IFS, which relaxes the dependency of membership and nonmembership value by $0 \leq \mu^{2}+\nu^{2} \leq 1$. Similarly, several generalizations of fuzzy sets are introduced for representing vagueness happening in different situations.

In 1999, Molodtsov [22] introduced soft sets and it is an adequate tool to handle vagueness in a parametric manner. A soft set is able to view as a bag with an approximate representation of the objects and involves two components that are predicate and approximate value set. As the initial description has an approximate nature, the machinery of traditional mathematics fails whereas the soft set can be used to manage many problems in this aspect. By considering this importance, several studies based on soft sets are done, and some of them can be seen in $[18,1,6,15]$. Later on, hybrid structures like fuzzy soft sets (FSS) [8], intuitionistic fuzzy soft set (IFSS) [19], Pythagorean fuzzy soft set (PFSS) [28] are introduced which can represent vagueness as well.

PFS is an extension of IFS. In contrast to IFS, PFS allows one to consign a membership value and a non-membership value resulting sum can exceed one. Studies on aggregation operations, decision-making problems, multi-criteria decision-making problems using PFS are appeared in papers of Garg et al. [9, 10]. Also, Peng et al. [27] give an extensive review of PFS. PFSS is a hybrid structure of the soft set and PFS, that can effectively deal with vagueness. Impressively PFSS grasp the properties of PFS and soft sets together. The structure, PFSS was established by Peng et al. [28]. After which Guleria et al. [12] gave the matrix representation, operation, and decision-making problems of PFSS. Later on, Athira et al. [4,5] studied the entropy and distance measure of PFSS. Recently, topological and group structures on PFSS are introduced and that can be seen in $[25,13]$ also Athira and Sunil proposed the incomplete PFSS [3]. 
As mentioned earlier, the similarity measure is a significant implement to estimate the degrees of association among two or more items. The idea of similarity measure for FS was initially introduced by Wang [31]. Meanwhile several studies were done on similarity measure of FS [14,33] and after which the similarity measure was extended to IFS [17], PFS [26] etc. Also, Majumdar and Samanta defined certain similarity measure for soft sets [21] and FSS [20]. Later on, it has been extended to IFSS [7]. Among various structures discussed so far, PFSS being the most generalized one. Vagueness can be represented more effectively by PFSS, and in this case, comparing their affinity is not readily available in the existing literature. This paves the way to the necessity of introducing similarity measures for PFSS.

This paper contains three sections besides the introduction. Section 2 includes basic definitions that are required for future study. In section 3, definitions of similarity measures and certain properties of the proposed measures are inserted. The final section consists of the applications of the new measures to the clustering algorithm as well as the benefits of proposed similarity measures with the already established measures.

\section{Preliminaries}

This section addresses elementary definitions beneficial for entire discussions. That is, the main definitions connected with PFS, soft sets, and PFSS. Unless otherwise specified, $\mho$ be the universal set, $\mathfrak{L}$ be parameter set, and $P(\mho)$ be the power set of $\mho$.

Definition 1 [34] A Pythagorean fuzzy set $\mathcal{P}$ on $\mho$ is the set $\left\{\left(u, \mu_{p}(u), \nu_{p}(u)\right)\right.$ : $u \in \mho\}$ where $\mu_{p}: \mho \rightarrow[0,1]$ and $\nu_{p}: \mho \rightarrow[0,1]$ with $0 \leq \mu_{p}^{2}+\nu_{p}^{2} \leq 1$.

Definition 2 [34] Let $p_{1}=\left(\mu_{1}, \nu_{1}\right)$ and $p_{2}=\left(\mu_{2}, \nu_{2}\right)$ be two Pythagorean fuzzy numbers.The set operations are given by;

1. $p_{1} \subseteq p_{2}$ if $\mu_{1} \leq \mu_{2} \& \nu_{1} \geq \nu_{2}$

2. $p_{1} \cup p_{2}=\left(\max \left\{\mu_{1}, \mu_{2}\right\}, \min \left\{\nu_{1}, \nu_{2}\right\}\right)$

3. $p_{1} \cap p_{2}=\left(\min \left\{\mu_{1}, \mu_{2}\right\}, \max \left\{\nu_{1}, \nu_{2}\right\}\right)$

4. $p^{c}=(\nu, \mu)$

Definition 3 [36] Let $P F S(\mho)$ be the set of all PFSs over $\mho$ and $\psi$ be a mapping $\psi: P F S(\mho) \times P F S(\mho) \rightarrow[0,1]$. Then $\psi$ is a similarity measure of PFSs if it holds:

1. $\left(\varphi_{1}, \varphi_{2}\right)=1 \Leftrightarrow \varphi_{1}=\varphi_{2}$

2. $\left(\varphi_{1}, \varphi_{2}\right)=\psi\left(\varphi_{2}, \varphi_{1}\right)$

3. $\left(\varphi_{1}, P_{3}\right) \leq \psi\left(\varphi_{1}, P_{2}\right) \wedge \psi\left(\varphi_{2}, \varphi_{3}\right)$ if $\varphi_{1} \subseteq \varphi_{2} \subseteq \varphi_{3}$

$\forall \varphi_{1}, \varphi_{2}, \varphi_{3} \in \operatorname{PFS}(\mho)$.

Definition 4 [22] The pair $(\mathcal{S}, \mathfrak{L})$ is soft set over $\mho$, if $\mathcal{S}$ is a mapping from $\mathfrak{L}$ to $P(\mho)$. 
Definition 5 [18] Let $\left(\mathcal{S}_{1}, \mathfrak{L}_{1}\right)$ and $\left(\mathcal{S}_{2}, \mathfrak{L}_{2}\right)$ are soft sets over $\mho$. We can define the set operations as follows;

1. $\left(\mathcal{S}_{1}, \mathfrak{L}_{1}\right) \widetilde{\widetilde{C}}\left(\mathcal{S}_{2}, \mathfrak{L}_{2}\right)$ if $\mathcal{S}_{1}(\mathfrak{h}) \subseteq \mathcal{S}_{2}(\mathfrak{h})$ and $\mathfrak{L}_{1} \subseteq \mathfrak{L}_{2}, \forall \mathfrak{h} \in \mathfrak{L}_{1}$. We say $\left(\mathcal{S}_{1}, \mathfrak{L}_{1}\right)$ and $\left(\mathcal{S}_{2}, \mathfrak{L}_{2}\right)$ are soft equal if $\left(\mathcal{S}_{1}, \mathfrak{L}_{1}\right) \widetilde{\subseteq}\left(\mathcal{S}_{2}, \mathfrak{L}_{2}\right)$ and $\left(\mathcal{S}_{2}, \mathfrak{L}_{2}\right) \widetilde{\subseteq}\left(\mathcal{S}_{1}, \mathfrak{L}_{1}\right)$.

2. The complement $\left(\left(\mathcal{S}_{1}, \mathfrak{L}_{1}\right)^{c}\right)$ of $\left(\mathcal{S}_{1}, \mathfrak{L}_{1}\right)$ is the soft set $\left(\mathcal{S}_{1}{ }^{c}, \mathfrak{L}_{1}\right)$, where $\mathcal{S}_{1}^{c}$ :

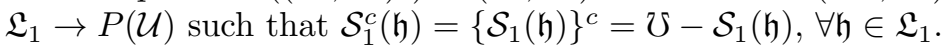

3. The union of $\left(\mathcal{S}_{1}, \mathfrak{L}_{1}\right)$ and $\left(\mathcal{S}_{2}, \mathfrak{L}_{2}\right)$ is denoted by $\left(\mathcal{S}_{1}, \mathfrak{L}_{1}\right) \widetilde{\cup}\left(\mathcal{S}_{2}, \mathfrak{L}_{2}\right)$ and is a soft set $\left(\mathcal{S}_{3}, \mathfrak{L}_{1} \cup \mathfrak{L}_{2}\right)$ where $\mathcal{S}_{3}: \mathfrak{L}_{1} \cup \mathfrak{L}_{2} \rightarrow P(\mho)$ is given by,

$$
\mathcal{S}_{3}(\mathfrak{h})=\left\{\begin{array}{l}
\mathcal{S}_{1}(\mathfrak{h}), \text { for } \mathfrak{h} \in \mathfrak{L}_{1}-\mathfrak{L}_{2} \\
\mathcal{S}_{2}(\mathfrak{h}), \text { for } \mathfrak{h} \in \mathfrak{L}_{2}-\mathfrak{L}_{1} \\
\mathcal{S}_{1}(\mathfrak{h}) \cup \mathcal{S}_{2}(\mathfrak{h}), \text { for } \mathfrak{h} \in \mathfrak{L}_{1} \cap \mathfrak{L}_{2}
\end{array} .\right.
$$

4. The intersection of $\left(\mathcal{S}_{1}, \mathfrak{L}_{1}\right)$ and $\left(\mathcal{S}_{2}, \mathfrak{L}_{2}\right)$ is denoted by $\left(\mathcal{S}_{1}, \mathfrak{L}_{1}\right) \widetilde{\cap}\left(\mathcal{S}_{2}, \mathfrak{L}_{2}\right)$ and is a soft set $\left(\mathcal{S}_{4}, \mathfrak{L}_{1} \cap \mathfrak{L}_{2}\right)$ where $\mathcal{S}_{4}: \mathfrak{L}_{1} \cap \mathfrak{L}_{2} \rightarrow P(\mho)$ is given by $\mathcal{S}_{4}(\mathfrak{h})=\mathcal{S}_{1}(\mathfrak{h}) \cap \mathcal{S}_{2}(\mathfrak{h}), \quad \forall \mathfrak{h} \in \mathfrak{L}_{1} \cap \mathfrak{L}_{2}$.

Definition 6 [16] Let $\mathrm{S}(\mho)$ denotes the collection of all soft sets over $\mho$. A similarity measure is a map $\psi: \mathrm{S}(\mho) \times \mathbf{S}(\mho) \rightarrow[0,1]$ with following properties; for $\left(\mathcal{S}_{1}, \mathfrak{L}\right),\left(\mathcal{S}_{2}, \mathfrak{L}\right),\left(\mathcal{S}_{3}, \mathfrak{L}\right) \in \mathrm{S}(\mho)$,

1. if $\left(\mathcal{S}_{1}, \mathfrak{L}\right)=\left(\mathcal{S}_{2}, \mathfrak{L}\right)$, then $\psi\left(\left(\mathcal{S}_{1}, \mathfrak{L}\right),\left(\mathcal{S}_{2}, \mathfrak{L}\right)\right)=1$

2. $\left.\quad\left(\mathcal{S}_{1}, \mathfrak{L}\right),\left(\mathcal{S}_{2}, \mathfrak{L}\right)\right)=\psi\left(\left(\mathcal{S}_{2}, \mathfrak{L}\right),\left(\mathcal{S}_{1}, \mathfrak{L}\right)\right)$

3. $\left.\left.\quad\left(\mathcal{S}_{1}, \mathfrak{L}\right),\left(\mathcal{S}_{3}, \mathfrak{L}\right)\right) \leq \psi\left(\mathcal{S}_{1}, \mathfrak{L}\right),\left(\mathcal{S}_{2}, \mathfrak{L}\right)\right)$ and $\left.\left.\left(\mathcal{S}_{1}, \mathfrak{L}\right),\left(\mathcal{S}_{3}, \mathfrak{L}\right)\right) \leq \psi\left(\mathcal{S}_{2}, \mathfrak{L}\right),\left(\mathcal{S}_{3}, \mathfrak{L}\right)\right)$ if $\left(\mathcal{S}_{1}, \mathfrak{L}\right) \widetilde{\subseteq}\left(\mathcal{S}_{2}, \mathfrak{L}\right) \widetilde{\subseteq}\left(\mathcal{S}_{3}, \mathfrak{L}\right)$.

Definition 7 [28] A PFSS over $\mho$ is a pair $(\mathcal{P}, \mathfrak{L})$ where $\mathfrak{L}$ is a collection of parameters and $\mathcal{P}$ is a mapping from $\mathfrak{L}$ into $\operatorname{PFS}(\mho)$ and $\operatorname{PFS}(\mho)$ is the set of all PFS subsets of $\mho$.

From the definition 7 it is clear that PFSS is a hybrid structure of PFS and soft set.

Definition 8 [28] Suppose $\mathfrak{L}_{1}, \mathfrak{L}_{2} \subseteq \mathfrak{L}$ and $\left(\mathcal{P}, \mathfrak{L}_{1}\right),\left(\mathcal{G}, \mathfrak{L}_{2}\right)$ are two PFSSs over $\mho$. $\left(\mathcal{P}, \mathfrak{L}_{1}\right)$ is said to be PFSS subset of $\left(\mathcal{P}, \mathfrak{L}_{2}\right)$ denoted as $\left(\mathcal{P}, \mathfrak{L}_{1}\right) \sqsubseteq\left(\mathcal{G}, \mathfrak{L}_{2}\right)$ if, 1. $\mathfrak{L}_{1} \subseteq \mathfrak{L}_{2}$

2. $\mathcal{P}(\mathfrak{h}) \subseteq \mathcal{G}(\mathfrak{h}), \forall \mathfrak{h} \in \mathfrak{L}_{1}$ i.e., $\forall x \in \mho \mathfrak{S}$ and $\mathfrak{h} \in \mathfrak{L}_{1}, \mu_{\mathcal{P}(\mathfrak{h})}(x) \leq \mu_{\mathcal{G}(\mathfrak{h})}(x)$ and $\nu_{\mathcal{P}(\mathfrak{h})}(x) \geq \nu_{\mathcal{G}(\mathfrak{h})}(x)$.

If $\left(\mathcal{P}, \mathfrak{L}_{1}\right) \sqsubseteq\left(\mathcal{G}, \mathfrak{L}_{2}\right)$ and $\left(\mathcal{G}, \mathfrak{L}_{2}\right) \sqsubseteq\left(\mathcal{P}, \mathfrak{L}_{1}\right)$ then $\left(\mathcal{P}, \mathfrak{L}_{1}\right)$ and $\left(\mathcal{G}, \mathfrak{L}_{2}\right)$ are said to be equal.

Definition 9 [28] The intersection of two PFSSs $\left(\mathcal{P}, \mathfrak{L}_{1}\right)$ and $\left(\mathcal{G}, \mathfrak{L}_{2}\right)$ over $\mho$, denoted by, $\left(\mathcal{P}, \mathfrak{L}_{1}\right) \sqcap\left(\mathcal{G}, \mathfrak{L}_{2}\right)$ and is defined to be the PFSS $\left(\mathcal{R}, \mathfrak{L}_{1} \cap \mathfrak{L}_{2}\right)$ where $\mathcal{R}$ is a map from $\mathfrak{L}_{1} \cap \mathfrak{L}_{2}$ into $\operatorname{PFS}(\mho)$ given by $\mathcal{R}(\mathfrak{h})=\mathcal{P}(\mathfrak{h}) \cap \mathcal{G}(\mathfrak{h})$, $\forall \mathfrak{h} \in \mathfrak{L}_{1} \cap \mathfrak{L}_{2}$, while the union of two PFSSs over $\mho$, denoted as $\left(\mathcal{P}, \mathfrak{L}_{1}\right) \sqcup\left(\mathcal{G}, \mathfrak{L}_{2}\right)$ and is defined to be $\left(\mathcal{T}, \mathfrak{L}_{1} \cup \mathfrak{L}_{2}\right)$ where $\mathcal{T}$ is a map from $\mathfrak{L}_{1} \cup \mathfrak{L}_{2}$ into PFS(ひ) given by,

$$
\mathcal{T}(\mathfrak{h})= \begin{cases}\mathcal{P}(\mathfrak{h}) & \text { for } \mathfrak{h} \in \mathfrak{L}_{1} \backslash \mathfrak{L}_{2} \\ \mathcal{G}(\mathfrak{h}) & \text { for } \mathfrak{h} \in \mathfrak{L}_{2} \backslash \mathfrak{L}_{1} \\ \mathcal{P}(\mathfrak{h}) \cup \mathcal{G}(\mathfrak{h}) & \text { for } \mathfrak{h} \in \mathfrak{L}_{1} \cap \mathfrak{L}_{2}\end{cases}
$$




\section{Similarity Measures of PFSS}

A real-valued function that measures out the similarity among two objects is called the similarity measure. This section explains the similarity measure for PFSSs, moreover different expressions to calculate similarity measures. Also, we proved a couple of theorems that describe certain interesting properties of the similarity measures.

The definition for similarity measure should emphasize the properties essential for a measure that quantifies the similarity between PFSSs. That is, the similarity measure between two PFSSs is maximum if and only if both are equal, and it is minimum if the sets are entirely different. To avoid unnecessary complexity we assume the values of similarity measures lie between 0 and 1 .

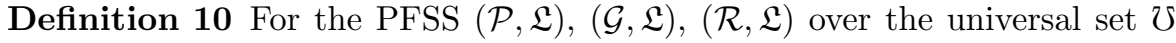
with parameter set $\mathfrak{L}$. A mapping $\Psi: P F S S(\mho) \times P F S S(\mho) \rightarrow \mathbb{R}^{+}$defines a similarity measure if it satisfies the four axioms given below,

A1. $0 \leq \Psi((\mathcal{P}, \mathfrak{L}),(\mathcal{G}, \mathfrak{L})) \leq 1$

A2. $\Psi((\mathcal{P}, \mathfrak{L}),(\mathcal{G}, \mathfrak{L}))=\Psi((\mathcal{G}, \mathfrak{L}),(\mathcal{P}, \mathfrak{L}))$

A3. $\Psi((\mathcal{P}, \mathfrak{L}),(\mathcal{G}, \mathfrak{L}))=1$ iff $(\mathcal{P}, \mathfrak{L})=(\mathcal{G}, \mathfrak{L})$

A4. $\Psi((\mathcal{P}, \mathfrak{L}),(\mathcal{R}, \mathfrak{L})) \leq \Psi((\mathcal{P}, \mathfrak{L}),(\mathcal{G}, \mathfrak{L}))$ and $\Psi((\mathcal{P}, \mathfrak{L}),(\mathcal{R}, \mathfrak{L})) \leq \Psi((\mathcal{G}, \mathfrak{L}),(\mathcal{R}, \mathfrak{L}))$ if $(\mathcal{P}, \mathfrak{L}) \sqsubseteq(\mathcal{G}, \mathfrak{L}) \sqsubseteq(\mathcal{R}, \mathfrak{L})$.

Theorem 1 Let $\mho=\left\{\mathfrak{u}_{1}, \mathfrak{u}_{2}, \cdots, \mathfrak{u}_{n}\right\}$ be universal set, $\mathfrak{L}=\left\{\mathfrak{h}_{1}, \mathfrak{h}_{2}, \cdots, \mathfrak{h}_{m}\right\}$ be the parameter set and $(\mathcal{P}, \mathfrak{L}),(\mathcal{G}, \mathfrak{L})$ are any two PFSSs over $\mathcal{\mho}$. Then $\Psi_{r}((\mathcal{P}, \mathfrak{L}),(\mathcal{G}, \mathfrak{L})), r=1,2,3,4,5,6,7,8,9,10$ are similarity measures.

1.

$$
\Psi_{1}((\mathcal{P}, \mathfrak{L}),(\mathcal{G}, \mathfrak{L}))=\frac{\sum_{i=1}^{n} \sum_{j=1}^{m}\left(\begin{array}{l}
\min \left\{\mu_{\mathcal{P}\left(\mathfrak{h}_{j}\right)}^{2}\left(\mathfrak{u}_{i}\right), \mu_{\mathcal{G}\left(\mathfrak{h}_{j}\right)}^{2}\left(\mathfrak{u}_{i}\right)\right\}+ \\
\min \left\{\nu_{\mathcal{P}\left(\mathfrak{h}_{j}\right)}^{2}\left(\mathfrak{u}_{i}\right), \nu_{\mathcal{G}\left(\mathfrak{h}_{j}\right)}^{2}\left(\mathfrak{u}_{i}\right)\right\}
\end{array}\right)}{\sum_{i=1}^{n} \sum_{j=1}^{m}\left(\begin{array}{l}
\max \left\{\mu_{\mathcal{P}\left(\mathfrak{h}_{j}\right)}^{2}\left(\mathfrak{u}_{i}\right), \mu_{\mathcal{G}\left(\mathfrak{h}_{j}\right)}^{2}\left(\mathfrak{u}_{i}\right)\right\}+ \\
\max \left\{\nu_{\mathcal{P}\left(\mathfrak{h}_{j}\right)}^{2}\left(\mathfrak{u}_{i}\right), \nu_{\mathcal{G}\left(\mathfrak{h}_{j}\right)}^{2}\left(\mathfrak{u}_{i}\right)\right\}
\end{array}\right)}
$$

2.

$$
\Psi_{2}((\mathcal{P}, \mathfrak{L}),(\mathcal{G}, \mathfrak{L}))=\frac{1}{m} \sum_{j=1}^{m} \frac{\sum_{i=1}^{n} 1-\min \left\{\begin{array}{l}
\left|\mu_{\mathcal{P}\left(\mathfrak{h}_{j}\right)}^{2}\left(u_{i}\right)-\mu_{\mathcal{G}\left(\mathfrak{h}_{j}\right)}^{2}\left(u_{i}\right)\right|, \\
\left|\nu_{\mathcal{P}\left(\mathfrak{h}_{j}\right)}^{2}\left(u_{i}\right)-\nu_{\mathcal{G}\left(\mathfrak{h}_{j}\right)}^{2}\left(u_{i}\right)\right|
\end{array}\right\}}{\sum_{i=1}^{n} 1+\max \left\{\begin{array}{l}
\left|\mu_{\mathcal{P}\left(\mathfrak{h}_{j}\right)}^{2}\left(u_{i}\right)-\mu_{\mathcal{G}\left(\mathfrak{h}_{j}\right)}^{2}\left(u_{i}\right)\right|, \\
\left|\nu_{\mathcal{P}\left(\mathfrak{h}_{j}\right)}^{2}\left(u_{i}\right)-\nu_{\mathcal{G}\left(\mathfrak{h}_{j}\right)}^{2}\left(u_{i}\right)\right|
\end{array}\right\}}
$$

3.

$$
\Psi_{3}((\mathcal{P}, \mathfrak{L}),(\mathcal{G}, \mathfrak{L}))=1-\frac{1}{2 m n} \sum_{j=1}^{m} \sum_{i=1}^{n}\left(\begin{array}{l}
\left|\mu_{\mathcal{P}\left(\mathfrak{h}_{j}\right)}^{2}\left(\mathfrak{u}_{i}\right)-\mu_{\mathcal{G}\left(\mathfrak{h}_{j}\right)}^{2}\left(\mathfrak{u}_{i}\right)\right|+ \\
\left|\nu_{\mathcal{P}\left(\mathfrak{h}_{j}\right)}^{2}\left(\mathfrak{u}_{i}\right)-\nu_{\mathcal{G}\left(\mathfrak{h}_{j}\right)}^{2}\left(\mathfrak{u}_{i}\right)\right|+ \\
\left|\pi_{\mathcal{P}\left(\mathfrak{h}_{j}\right)}^{2}\left(\mathfrak{u}_{i}\right)-\pi_{\mathcal{G}\left(\mathfrak{h}_{j}\right)}^{2}\left(\mathfrak{u}_{i}\right)\right|
\end{array}\right)
$$

4.

$$
\Psi_{4}((\mathcal{P}, \mathfrak{L}),(\mathcal{G}, \mathfrak{L}))=\frac{1}{m n} \sum_{j=1}^{m} \sum_{i=1}^{n} \cos \left[\frac{\pi}{2} \max \left\{\begin{array}{l}
\left|\mu_{\mathcal{P}\left(\mathfrak{h}_{j}\right)}^{2}\left(\mathfrak{u}_{i}\right)-\mu_{\mathcal{G}\left(\mathfrak{h}_{j}\right)}^{2}\left(\mathfrak{u}_{i}\right)\right|, \\
\left.\left|\nu_{\mathcal{P}\left(\mathfrak{h}_{j}\right)}^{2}\left(\mathfrak{u}_{i}\right)-\nu_{\mathcal{G}\left(\mathfrak{h}_{j}\right)}^{2}\left(\mathfrak{u}_{i}\right)\right|\right\}
\end{array}\right]\right.
$$


5.

$$
\Psi_{5}((\mathcal{P}, \mathfrak{L}),(\mathcal{G}, \mathfrak{L}))=\frac{1}{m n} \sum_{j=1}^{m} \sum_{i=1}^{n} \cos \left[\frac{\pi}{4}\left(\begin{array}{l}
\left|\mu_{\mathcal{P}\left(\mathfrak{h}_{j}\right)}^{2}\left(\mathfrak{u}_{i}\right)-\mu_{\mathcal{G}\left(\mathfrak{h}_{j}\right)}^{2}\left(\mathfrak{u}_{i}\right)\right| \\
+\left|\nu_{\mathcal{P}\left(\mathfrak{h}_{j}\right)}^{2}\left(\mathfrak{u}_{i}\right)-\nu_{\mathcal{G}\left(\mathfrak{h}_{j}\right)}^{2}\left(\mathfrak{u}_{i}\right)\right|
\end{array}\right)\right]
$$

6.

$$
\Psi_{6}((\mathcal{P}, \mathfrak{L}),(\mathcal{G}, \mathfrak{L}))=1-\frac{1}{m} \sum_{j=1}^{m}\left(\begin{array}{l}
\sum_{i=1}^{n}\left|\mu_{\mathcal{P}\left(\mathfrak{h}_{j}\right)}^{2}\left(\mathfrak{u}_{i}\right)-\mu_{\mathcal{G}\left(\mathfrak{h}_{j}\right)}^{2}\left(\mathfrak{u}_{i}\right)\right| \\
+\left|\nu_{\mathcal{P}\left(\mathfrak{h}_{j}\right)}^{2}\left(\mathfrak{u}_{i}\right)-\nu_{\mathcal{G}\left(\mathfrak{h}_{j}\right)}^{2}\left(\mathfrak{u}_{i}\right)\right| \\
\sum_{i=1}^{n}\left|\mu_{\mathcal{P}\left(\mathfrak{h}_{j}\right)}^{2}\left(\mathfrak{u}_{i}\right)+\mu_{\mathcal{G}\left(\mathfrak{h}_{j}\right)}^{2}\left(\mathfrak{u}_{i}\right)\right| \\
+\left|\nu_{\mathcal{P}\left(\mathfrak{h}_{j}\right)}^{2}\left(\mathfrak{u}_{i}\right)+\nu_{\mathcal{G}\left(\mathfrak{h}_{j}\right)}^{2}\left(\mathfrak{u}_{i}\right)\right|
\end{array}\right)
$$

7.

$$
\Psi_{7}((\mathcal{P}, \mathfrak{L}),(\mathcal{G}, \mathfrak{L}))=\frac{1}{m} \sum_{j=1}^{m}\left(\begin{array}{l}
\sum_{i=1}^{n} \min \left\{\mu_{\mathcal{P}\left(\mathfrak{h}_{j}\right)}^{2}, \mu_{\mathcal{G}\left(\mathfrak{h}_{j}\right)}^{2}\right\}+ \\
\frac{\min \left\{1-\nu_{\mathcal{P}\left(\mathfrak{h}_{j}\right)}^{2}, 1-\nu_{\mathcal{G}\left(\mathfrak{h}_{j}\right)}^{2}\right\}}{\sum_{i=1}^{n} \max \left\{\mu_{\mathcal{P}\left(\mathfrak{h}_{j}\right)}^{2}, \mu_{\mathcal{G}\left(\mathfrak{h}_{j}\right)}^{2}\right\}+} \\
\max \left\{1-\nu_{\mathcal{P}\left(\mathfrak{h}_{j}\right)}^{2}, 1-\nu_{\mathcal{G}\left(\mathfrak{h}_{j}\right)}^{2}\right\}
\end{array}\right)
$$

8.

$$
\Psi_{8}((\mathcal{P}, \mathfrak{L}),(\mathcal{G}, \mathfrak{L}))=\left(\begin{array}{c}
\frac{\alpha}{m} \sum_{j=1}^{m} \frac{\sum_{i=1}^{n} \min \left\{\mu_{\mathcal{P}\left(\mathfrak{h}_{j}\right)}^{2}, \mu_{\mathcal{G}\left(\mathfrak{h}_{j}\right)}^{2}\right\}}{\sum_{i=1}^{n} \max \left\{\mu_{\mathcal{P}\left(\mathfrak{h}_{j}\right)}^{2}, \mu_{\mathcal{G}\left(\mathfrak{h}_{j}\right)}^{2}\right\}}+ \\
\frac{1-\alpha}{m} \sum_{j=1}^{m} \frac{\sum_{i=1}^{n} \min \left\{\nu_{\mathcal{P}\left(\mathfrak{h}_{j}\right)}^{2}, \nu_{\mathcal{G}\left(\mathfrak{h}_{j}\right)}^{2}\right\}}{\sum_{i=1}^{n} \max \left\{\nu_{\mathcal{P}\left(\mathfrak{h}_{j}\right)}^{2}, \nu_{\mathcal{G}\left(\mathfrak{h}_{j}\right)}^{2}\right\}}
\end{array}\right), \alpha \in[0,1]
$$

9.

$$
\Psi_{9}((\mathcal{P}, \mathfrak{L}),(\mathcal{G}, \mathfrak{L}))=\frac{1}{m} \sum_{j=1}^{m} \frac{\sum_{i=1}^{n}\left(\begin{array}{l}
\mu_{\mathcal{P}\left(\mathfrak{h}_{j}\right)}^{2}\left(\mathfrak{u}_{i}\right) \cdot \mu_{\mathcal{G}\left(\mathfrak{h}_{j}\right)}^{2}\left(\mathfrak{u}_{i}\right)+\nu_{\mathcal{P}\left(\mathfrak{h}_{j}\right)}^{2}\left(\mathfrak{u}_{i}\right) \cdot \nu_{\mathcal{G}\left(\mathfrak{h}_{j}\right)}^{2}\left(\mathfrak{u}_{i}\right) \\
+\pi_{\mathcal{P}\left(\mathfrak{h}_{j}\right)}^{2}\left(\mathfrak{u}_{i}\right) \cdot \pi_{\mathcal{G}\left(\mathfrak{h}_{j}\right)}^{2}\left(\mathfrak{u}_{i}\right)
\end{array}\right)}{\max \left\{\begin{array}{l}
\sum_{i=1}^{n}\left(\mu_{\mathcal{P}\left(\mathfrak{h}_{j}\right)}^{4}\left(\mathfrak{u}_{i}\right)+\nu_{\mathcal{G}\left(\mathfrak{h}_{j}\right)}^{4}\left(\mathfrak{u}_{i}\right)+\pi_{\mathcal{P}\left(\mathfrak{h}_{j}\right)}^{4}\left(\mathfrak{u}_{i}\right)\right), \\
\sum_{i=1}^{n}\left(\mu_{\mathcal{G}\left(\mathfrak{h}_{j}\right)}^{4}\left(\mathfrak{u}_{i}\right)+\nu_{\mathcal{G}\left(\mathfrak{h}_{j}\right)}^{4}\left(\mathfrak{u}_{i}\right)+\pi_{\mathcal{G}\left(\mathfrak{h}_{j}\right)}^{4}\left(\mathfrak{u}_{i}\right)\right)
\end{array}\right\}}
$$

10.

$$
\Psi_{10}((\mathcal{P}, \mathfrak{L}),(\mathcal{G}, \mathfrak{L}))=1-\frac{1}{m} \sum_{j=1}^{m}\left(\begin{array}{c}
\sum_{i=1}^{n}\left(\left|\mu_{\mathcal{P}\left(\mathfrak{h}_{j}\right)}^{2}\left(\mathfrak{u}_{i}\right)-\mu_{\mathcal{G}\left(\mathfrak{h}_{j}\right)}^{2}\left(\mathfrak{u}_{i}\right)\right|^{2}+\right. \\
\left|\nu_{\mathcal{P}\left(\mathfrak{h}_{j}\right)}^{2}\left(\mathfrak{u}_{i}\right)-\nu_{\mathcal{G}\left(\mathfrak{h}_{j}\right)}^{2}\left(\mathfrak{u}_{i}\right)\right|^{2}+ \\
\frac{\left.\left|\pi_{\mathcal{P}\left(\mathfrak{h}_{j}\right)}^{2}\left(\mathfrak{u}_{i}\right)-\pi_{\mathcal{G}\left(\mathfrak{h}_{j}\right)}^{2}\left(\mathfrak{u}_{i}\right)\right|^{2}\right)}{\sum_{i=1}^{n}\left(\left|\mu_{\mathcal{P}\left(\mathfrak{h}_{j}\right)}^{2}\left(\mathfrak{u}_{i}\right)+\mu_{\mathcal{G}\left(\mathfrak{h}_{j}\right)}^{2}\left(\mathfrak{u}_{i}\right)\right|^{2}+\right.} \\
\left|\nu_{\mathcal{P}\left(\mathfrak{h}_{j}\right)}^{2}\left(\mathfrak{u}_{i}\right)+\nu_{\mathcal{G}\left(\mathfrak{h}_{j}\right)}^{2}\left(\mathfrak{u}_{i}\right)\right|^{2}+ \\
\left.\left|\pi_{\mathcal{P}\left(\mathfrak{h}_{j}\right)}^{2}\left(\mathfrak{u}_{i}\right)+\pi_{\mathcal{G}\left(\mathfrak{h}_{j}\right)}^{2}\left(\mathfrak{u}_{i}\right)\right|^{2}\right)
\end{array}\right)^{\frac{1}{2}}
$$


Proof For proving $\Psi_{r}((\mathcal{P}, \mathfrak{L}),(\mathcal{G}, \mathfrak{L})), r=1,2, \cdots, 10$ are similarity measure, it must satisfy the four axioms $A_{1}, A_{2}, A_{3}$ and $A_{4}$. We will prove axiom $A_{3}$ for $\Psi_{9}((\mathcal{P}, \mathfrak{L}),(\mathcal{G}, \mathfrak{L}))$, axiom $A_{4}$ for $\Psi_{r}((\mathcal{P}, \mathfrak{L}),(\mathcal{G}, \mathfrak{L})), r=3,4,6$ and rest of the proof is straight forward.

For proving Axiom $A_{3}$ for $\Psi_{9}((\mathcal{P}, \mathfrak{L}),(\mathcal{G}, \mathfrak{L}))$, let $\Psi_{9}((\mathcal{P}, \mathfrak{L}),(\mathcal{G}, \mathfrak{L}))=1$ implies that

$$
\frac{1}{m} \sum_{j=1}^{m} \frac{\sum_{i=1}^{n}\left(\begin{array}{l}
\mu_{\mathcal{P}\left(\mathfrak{h}_{j}\right)}^{2}\left(\mathfrak{u}_{i}\right) \cdot \mu_{\mathcal{G}\left(\mathfrak{h}_{j}\right)}^{2}\left(\mathfrak{u}_{i}\right)+\nu_{\mathcal{P}\left(\mathfrak{h}_{j}\right)}^{2}\left(\mathfrak{u}_{i}\right) \cdot \nu_{\mathcal{G}\left(\mathfrak{h}_{j}\right)}^{2}\left(\mathfrak{u}_{i}\right) \\
+\pi_{\mathcal{P}\left(\mathfrak{h}_{j}\right)}^{2}\left(\mathfrak{u}_{i}\right) \cdot \pi_{\mathcal{G}\left(\mathfrak{h}_{j}\right)}^{2}\left(\mathfrak{u}_{i}\right)
\end{array}\right)}{\max \left\{\begin{array}{l}
\sum_{i=1}^{n}\left(\mu_{\mathcal{P}\left(\mathfrak{h}_{j}\right)}^{4}\left(\mathfrak{u}_{i}\right)+\nu_{\mathcal{P}\left(\mathfrak{h}_{j}\right)}^{4}\left(\mathfrak{u}_{i}\right)+\pi_{\mathcal{P}\left(\mathfrak{h}_{j}\right)}^{4}\left(\mathfrak{u}_{i}\right)\right), \\
\sum_{i=1}^{n}\left(\mu_{\mathcal{G}\left(\mathfrak{h}_{j}\right)}^{4}\left(\mathfrak{u}_{i}\right)+\nu_{\mathcal{G}\left(\mathfrak{h}_{j}\right)}^{4}\left(\mathfrak{u}_{i}\right)+\pi_{\mathcal{G}\left(\mathfrak{h}_{j}\right)}^{4}\left(\mathfrak{u}_{i}\right)\right)
\end{array}\right\}}=1
$$

Then for each $j=1,2, \cdots, m$,

$$
\begin{gathered}
\sum_{i=1}^{n}\left(\begin{array}{l}
\mu_{\mathcal{P}\left(\mathfrak{h}_{j}\right)}^{2}\left(\mathfrak{u}_{i}\right) \cdot \mu_{\mathcal{G}\left(\mathfrak{h}_{j}\right)}^{2}\left(\mathfrak{u}_{i}\right)+\nu_{\mathcal{P}\left(\mathfrak{h}_{j}\right)}^{2}\left(\mathfrak{u}_{i}\right) \cdot \nu_{\mathcal{G}\left(\mathfrak{h}_{j}\right)}^{2}\left(\mathfrak{u}_{i}\right) \\
+\pi_{\mathcal{P}\left(\mathfrak{h}_{j}\right)}^{2}\left(\mathfrak{u}_{i}\right) \cdot \pi_{\mathcal{G}\left(\mathfrak{h}_{j}\right)}^{2}\left(\mathfrak{u}_{i}\right)
\end{array}\right) \\
=\max \left\{\begin{array}{l}
\sum_{i=1}^{n}\left(\mu_{\mathcal{P}\left(\mathfrak{h}_{j}\right)}^{4}\left(\mathfrak{u}_{i}\right)+\nu_{\mathcal{P}\left(\mathfrak{h}_{j}\right)}^{4}\left(\mathfrak{u}_{i}\right)+\pi_{\mathcal{P}\left(\mathfrak{h}_{j}\right)}^{4}\left(\mathfrak{u}_{i}\right)\right), \\
\sum_{i=1}^{n}\left(\mu_{\mathcal{G}\left(\mathfrak{h}_{j}\right)}^{4}\left(\mathfrak{u}_{i}\right)+\nu_{\mathcal{G}\left(\mathfrak{h}_{j}\right)}^{4}\left(\mathfrak{u}_{i}\right)+\pi_{\mathcal{G}\left(\mathfrak{h}_{j}\right)}^{4}\left(\mathfrak{u}_{i}\right)\right)
\end{array}\right\} \\
\Longrightarrow \vec{V}_{1} \cdot \vec{V}_{2}=\max \left\{\left(\vec{V}_{1} \cdot \vec{V}_{1}\right)^{2}, \quad\left(\vec{V}_{2} \cdot \vec{V}_{2}\right)^{2}\right\}
\end{gathered}
$$

where,

$\vec{V}_{1}=\left(\mu_{\mathcal{P}\left(\mathfrak{h}_{j}\right)}^{2}\left(u_{1}\right), \ldots, \mu_{\mathcal{P}\left(\mathfrak{h}_{j}\right)}^{2}\left(u_{n}\right), \nu_{\mathcal{P}\left(\mathfrak{h}_{j}\right)}^{2}\left(u_{1}\right), \ldots, \nu_{\mathcal{P}\left(\mathfrak{h}_{j}\right)}^{2}\left(u_{n}\right), \pi_{\mathcal{P}\left(\mathfrak{h}_{j}\right)}^{2}\left(u_{1}\right), \ldots, \pi_{\mathcal{P}\left(\mathfrak{h}_{j}\right)}^{2}\left(u_{n}\right)\right)$ $\overrightarrow{V_{2}}=\left(\mu_{\mathcal{G}\left(\mathfrak{h}_{j}\right)}^{2}\left(u_{1}\right), \ldots, \mu_{\mathcal{G}\left(\mathfrak{h}_{j}\right)}^{2}\left(u_{n}\right), \nu_{\mathcal{G}\left(\mathfrak{h}_{j}\right)}^{2}\left(u_{1}\right), \ldots, \nu_{\mathcal{G}\left(\mathfrak{h}_{j}\right)}^{2}\left(u_{n}\right), \pi_{\mathcal{G}\left(\mathfrak{h}_{j}\right)}^{2}\left(u_{1}\right), \ldots, \pi_{\mathcal{G}\left(\mathfrak{h}_{j}\right)}^{2}\left(u_{n}\right)\right)$. The Cauchy Schwarz inequality for positive real numbers gives, $\overrightarrow{V_{1}} \cdot \overrightarrow{V_{2}} \leq\left(\vec{V}_{1} \cdot \vec{V}_{1}\right) \cdot\left(\vec{V}_{2} \cdot \vec{V}_{2}\right) \leq \max \left\{\left(\vec{V}_{1} \cdot \vec{V}_{1}\right)^{2}, \quad\left(\vec{V}_{2} \cdot \vec{V}_{2}\right)^{2}\right\}$ and the equality retains iff $\vec{V}_{1}=c \overrightarrow{V_{2}}$ for a constant $c$.

Thus from equation 11 we get, $\left(\sum_{i=1}^{n} \mu_{\mathcal{P}\left(\mathfrak{h}_{j}\right)}^{2}\left(\mathfrak{u}_{i}\right), \sum_{i=1}^{n} \nu_{\mathcal{P}\left(\mathfrak{h}_{j}\right)}^{2}\left(\mathfrak{u}_{i}\right), \sum_{i=1}^{n} \pi_{\mathcal{P}\left(\mathfrak{h}_{j}\right)}^{2}\left(\mathfrak{u}_{i}\right)\right)=$ $c\left(\sum_{i=1}^{n} \mu_{\mathcal{G}\left(\mathfrak{h}_{j}\right)}^{2}\left(\mathfrak{u}_{i}\right), \sum_{i=1}^{n} \nu_{\mathcal{G}\left(\mathfrak{h}_{j}\right)}^{2}\left(\mathfrak{u}_{i}\right), \sum_{i=1}^{n} \pi_{\mathcal{G}\left(\mathfrak{h}_{j}\right)}^{2}\left(\mathfrak{u}_{i}\right)\right)$ and that directly implies that $c=1$. Thus $\vec{V}_{1}=\vec{V}_{2}$.

Now we are going to prove axiom $A_{4}$. So consider $(\mathcal{P}, \mathfrak{L}) \sqsubseteq(\mathcal{G}, \mathfrak{L} \sqsubseteq$ $(\mathcal{R}, \mathfrak{L})$. It implies that $\mu_{\mathcal{P}\left(\mathfrak{h}_{j}\right)}\left(\mathfrak{u}_{i}\right) \leq \mu_{\mathcal{G}\left(\mathfrak{h}_{j}\right)}\left(\mathfrak{u}_{i}\right) \leq \mu_{\mathcal{R}\left(\mathfrak{h}_{j}\right)}\left(\mathfrak{u}_{i}\right)$ and $\nu_{\mathcal{P}\left(\mathfrak{h}_{j}\right)}\left(\mathfrak{u}_{i}\right) \geq$ $\nu_{\mathcal{G}\left(\mathfrak{h}_{j}\right)}\left(\mathfrak{u}_{i}\right) \geq \nu_{\mathcal{R}\left(\mathfrak{h}_{j}\right)}\left(\mathfrak{u}_{i}\right) \quad \forall i, j$.

1.

$$
\Psi_{3}((\mathcal{P}, \mathfrak{L}),(\mathcal{G}, \mathfrak{L}))=1-\frac{1}{2 m n} \sum_{j=1}^{m} \sum_{i=1}^{n}\left(\begin{array}{l}
\left|\mu_{\mathcal{P}\left(\mathfrak{h}_{j}\right)}^{2}\left(\mathfrak{u}_{i}\right)-\mu_{\mathcal{G}\left(\mathfrak{h}_{j}\right)}^{2}\left(\mathfrak{u}_{i}\right)\right|+ \\
\left|\nu_{\mathcal{P}\left(\mathfrak{h}_{j}\right)}^{2}\left(\mathfrak{u}_{i}\right)-\nu_{\mathcal{G}\left(\mathfrak{h}_{j}\right)}^{2}\left(\mathfrak{u}_{i}\right)\right|+ \\
\left|\pi_{\mathcal{P}\left(\mathfrak{h}_{j}\right)}^{2}\left(\mathfrak{u}_{i}\right)-\pi_{\mathcal{G}\left(\mathfrak{h}_{j}\right)}^{2}\left(\mathfrak{u}_{i}\right)\right|
\end{array}\right)
$$




$$
\Psi_{3}((\mathcal{P}, \mathfrak{L}),(\mathcal{R}, \mathfrak{L}))=1-\frac{1}{2 m n} \sum_{j=1}^{m} \sum_{i=1}^{n}\left(\begin{array}{l}
\left|\mu_{\mathcal{P}\left(\mathfrak{h}_{j}\right)}^{2}\left(\mathfrak{u}_{i}\right)-\mu_{\mathcal{R}\left(\mathfrak{h}_{j}\right)}^{2}\left(\mathfrak{u}_{i}\right)\right|+ \\
\left|\nu_{\mathcal{P}\left(\mathfrak{h}_{j}\right)}^{2}\left(\mathfrak{u}_{i}\right)-\nu_{\mathcal{R}\left(\mathfrak{h}_{j}\right)}^{2}\left(\mathfrak{u}_{i}\right)\right|+ \\
\left|\pi_{\mathcal{P}\left(\mathfrak{h}_{j}\right)}^{2}\left(\mathfrak{u}_{i}\right)-\pi_{\mathcal{R}\left(\mathfrak{h}_{j}\right)}^{2}\left(\mathfrak{u}_{i}\right)\right|
\end{array}\right)
$$

Let $\Psi_{3}((\mathcal{P}, \mathfrak{L}),(\mathcal{G}, \mathfrak{L}))-\Psi_{3}((\mathcal{P}, \mathfrak{L}),(\mathcal{R}, \mathfrak{L}))=\Delta \Psi_{3}$.

$$
\begin{aligned}
& \Delta \Psi_{3}= \frac{1}{2 m n} \sum_{j=1}^{m} \sum_{i=1}^{n}\left(\begin{array}{l}
\left|\mu_{\mathcal{P}\left(\mathfrak{h}_{j}\right)}^{2}\left(\mathfrak{u}_{i}\right)-\mu_{\mathcal{R}\left(\mathfrak{h}_{j}\right)}^{2}\left(\mathfrak{u}_{i}\right)\right|+\left|\nu_{\mathcal{P}\left(\mathfrak{h}_{j}\right)}^{2}\left(\mathfrak{u}_{i}\right)-\nu_{\mathcal{R}\left(\mathfrak{h}_{j}\right)}^{2}\left(\mathfrak{u}_{i}\right)\right|+ \\
\left|\pi_{\mathcal{P}\left(\mathfrak{h}_{j}\right)}^{2}\left(\mathfrak{u}_{i}\right)-\pi_{\mathcal{R}\left(\mathfrak{h}_{j}\right)}^{2}\left(\mathfrak{u}_{i}\right)\right|-\left|\mu_{\mathcal{P}\left(\mathfrak{h}_{j}\right)}^{2}\left(\mathfrak{u}_{i}\right)-\mu_{\mathcal{G}\left(\mathfrak{h}_{j}\right)}^{2}\left(\mathfrak{u}_{i}\right)\right|- \\
\left|\nu_{\mathcal{P}\left(\mathfrak{h}_{j}\right)}^{2}\left(\mathfrak{u}_{i}\right)-\nu_{\mathcal{G}\left(\mathfrak{h}_{j}\right)}^{2}\left(\mathfrak{u}_{i}\right)\right|-\left|\pi_{\mathcal{P}\left(\mathfrak{h}_{j}\right)}^{2}\left(\mathfrak{u}_{i}\right)-\pi_{\mathcal{G}\left(\mathfrak{h}_{j}\right)}^{2}\left(\mathfrak{u}_{i}\right)\right|
\end{array}\right) \\
&=\frac{1}{2 m n} \sum_{j=1}^{m} \sum_{i=1}^{n}\left(\begin{array}{l}
\mu_{\mathcal{R}\left(\mathfrak{h}_{j}\right)}^{2}\left(\mathfrak{u}_{i}\right)-\mu_{\mathcal{G}\left(\mathfrak{h}_{j}\right)}^{2}\left(\mathfrak{u}_{i}\right)+\nu_{\mathcal{G}\left(\mathfrak{h}_{j}\right)}^{2}\left(\mathfrak{u}_{i}\right)-\nu_{\mathcal{R}\left(\mathfrak{h}_{j}\right)}^{2}\left(\mathfrak{u}_{i}\right)+ \\
\mid \mu_{\mathcal{P}\left(\mathfrak{h}_{j}\right)}^{2}\left(\mathfrak{u}_{i}\right)+\nu_{\mathcal{P}\left(\mathfrak{h}_{j}\right)}^{2}\left(\mathfrak{u}_{i}\right)-\left(\mu_{\mathcal{R}\left(\mathfrak{h}_{j}\right)}^{2}\left(\mathfrak{u}_{i}\right)+\nu_{\mathcal{R}\left(\mathfrak{h}_{j}\right)}^{2}\left(\mathfrak{u}_{i}\right) \mid-\right. \\
\left.\mid \mu_{\mathcal{P}\left(\mathfrak{h}_{j}\right)}^{2}\left(\mathfrak{u}_{i}\right)+\nu_{\mathcal{P}\left(\mathfrak{h}_{j}\right)}^{2}\right)\left(\mathfrak{u}_{i}\right)-\left(\mu_{\mathcal{G}\left(\mathfrak{h}_{j}\right)}^{2}\right)\left(\mathfrak{u}_{i}\right)+\nu_{\mathcal{G}\left(\mathfrak{h}_{j}\right)}^{2}\left(\mathfrak{u}_{i}\right) \mid
\end{array}\right)
\end{aligned}
$$

Consider four cases;

Case 1: $\mu_{\mathcal{P}\left(\mathfrak{h}_{j}\right)}^{2}\left(\mathfrak{u}_{i}\right)+\nu_{\mathcal{P}\left(\mathfrak{h}_{j}\right)}^{2}\left(\mathfrak{u}_{i}\right) \geq \max \left\{\mu_{\mathcal{R}\left(\mathfrak{h}_{j}\right)}^{2}\left(\mathfrak{u}_{i}\right)+\nu_{\mathcal{R}\left(\mathfrak{h}_{j}\right)}^{2}\left(\mathfrak{u}_{i}\right), \mu_{\mathcal{G}\left(\mathfrak{h}_{j}\right)}^{2}\left(\mathfrak{u}_{i}\right)+\right.$ $\left.\nu_{\mathcal{G}\left(\mathfrak{h}_{j}\right)}^{2}\left(\mathfrak{u}_{i}\right)\right\}$

then $\Delta \Psi_{3}=\frac{1}{2 m n} \sum_{j=1}^{m} \sum_{i=1}^{n}\left(\nu_{\mathcal{G}\left(\mathfrak{h}_{j}\right)}^{2}\left(\mathfrak{u}_{i}\right)-\nu_{\mathcal{R}\left(\mathfrak{h}_{j}\right)}^{2}\left(\mathfrak{u}_{i}\right)\right) \geq 0$.

Case 2: $\mu_{\mathcal{P}\left(\mathfrak{h}_{j}\right)}^{2}\left(\mathfrak{u}_{i}\right)+\nu_{\mathcal{P}\left(\mathfrak{h}_{j}\right)}^{2}\left(\mathfrak{u}_{i}\right) \leq \min \left\{\mu_{\mathcal{R}\left(\mathfrak{h}_{j}\right)}^{2}\left(\mathfrak{u}_{i}\right)+\nu_{\mathcal{R}\left(\mathfrak{h}_{j}\right)}^{2}\left(\mathfrak{u}_{i}\right), \mu_{\mathcal{G}\left(\mathfrak{h}_{j}\right)}^{2}\left(\mathfrak{u}_{i}\right)+\right.$ $\left.\nu_{\mathcal{G}\left(\mathfrak{h}_{j}\right)}^{2}\left(\mathfrak{u}_{i}\right)\right\}$

then $\Delta \Psi_{3}=\frac{1}{2 m n} \sum_{j=1}^{m} \sum_{i=1}^{n} 2\left(\mu_{\mathcal{R}\left(\mathfrak{h}_{j}\right)}^{2}\left(\mathfrak{u}_{i}\right)-\mu_{\mathcal{G}\left(\mathfrak{h}_{j}\right)}^{2}\left(\mathfrak{u}_{i}\right)\right) \geq 0$.

Case 3: $\mu_{\mathcal{G}\left(\mathfrak{h}_{j}\right)}^{2}\left(\mathfrak{u}_{i}\right)+\nu_{\mathcal{G}\left(\mathfrak{h}_{j}\right)}^{2}\left(\mathfrak{u}_{i}\right) \leq \mu_{\mathcal{P}\left(\mathfrak{h}_{j}\right)}^{2}\left(\mathfrak{u}_{i}\right)+\nu_{\mathcal{P}\left(\mathfrak{h}_{j}\right)}^{2}\left(\mathfrak{u}_{i}\right) \leq \mu_{\mathcal{R}\left(\mathfrak{h}_{j}\right)}^{2}\left(\mathfrak{u}_{i}\right)+$ $\nu_{\mathcal{R}\left(\mathfrak{h}_{j}\right)}^{2}\left(\mathfrak{u}_{i}\right)$ then,

$\Delta \Psi_{3}=\frac{1}{2 m n} \sum_{j=1}^{m} \sum_{i=1}^{n} 2\left(\mu_{\mathcal{R}\left(\mathfrak{h}_{j}\right)}^{2}\left(\mathfrak{u}_{i}\right)+\nu_{\mathcal{G}\left(\mathfrak{h}_{j}\right)}^{2}\left(\mathfrak{u}_{i}\right)-\mu_{\mathcal{P}\left(\mathfrak{h}_{j}\right)}^{2}\left(\mathfrak{u}_{i}\right)-\nu_{\mathcal{P}\left(\mathfrak{h}_{j}\right)}^{2}\left(\mathfrak{u}_{i}\right)\right)$

$\geq \frac{1}{2 m n} \sum_{j=1}^{m} \sum_{i=1}^{n} 2\left(\begin{array}{l}{\left[\mu_{\mathcal{P}\left(\mathfrak{h}_{j}\right)}^{2}\left(\mathfrak{u}_{i}\right)+\nu_{\mathcal{P}\left(\mathfrak{h}_{j}\right)}^{2}\left(\mathfrak{u}_{i}\right)-\nu_{\mathcal{R}\left(\mathfrak{h}_{j}\right)}^{2}\left(\mathfrak{u}_{i}\right)\right]+} \\ \nu_{\mathcal{G}\left(\mathfrak{h}_{j}\right)}^{2}\left(\mathfrak{u}_{i}\right)-\mu_{\mathcal{P}\left(\mathfrak{h}_{j}\right)}^{2}\left(\mathfrak{u}_{i}\right)-\nu_{\mathcal{P}\left(\mathfrak{h}_{j}\right)}^{2}\left(\mathfrak{u}_{i}\right)\end{array}\right)$

$=\frac{1}{2 m n} \sum_{j=1}^{m} \sum_{i=1}^{n} 2\left(\nu_{\mathcal{G}\left(\mathfrak{h}_{j}\right)}^{2}\left(\mathfrak{u}_{i}\right)-\nu_{\mathcal{R}\left(\mathfrak{h}_{j}\right)}^{2}\left(\mathfrak{u}_{i}\right)\right) \geq 0$.

Case 4: $\mu_{\mathcal{R}\left(\mathfrak{h}_{j}\right)}^{2}\left(\mathfrak{u}_{i}\right)+\nu_{\mathcal{R}\left(\mathfrak{h}_{j}\right)}^{2}\left(\mathfrak{u}_{i}\right) \leq \mu_{\mathcal{P}\left(\mathfrak{h}_{j}\right)}^{2}\left(\mathfrak{u}_{i}\right)+\nu_{\mathcal{P}\left(\mathfrak{h}_{j}\right)}^{2}\left(\mathfrak{u}_{i}\right) \leq \mu_{\mathcal{G}\left(\mathfrak{h}_{j}\right)}^{2}\left(\mathfrak{u}_{i}\right)+$ $\nu_{\mathcal{G}\left(\mathfrak{h}_{j}\right)}^{2}\left(\mathfrak{u}_{i}\right)$ then

$\Delta \Psi_{3}=\frac{1}{2 m n} \sum_{j=1}^{m} \sum_{i=1}^{n} 2\left(\mu_{\mathcal{P}\left(\mathfrak{h}_{j}\right)}^{2}\left(\mathfrak{u}_{i}\right)+\nu_{\mathcal{P}\left(\mathfrak{h}_{j}\right)}^{2}\left(\mathfrak{u}_{i}\right)-\nu_{\mathcal{R}\left(\mathfrak{h}_{j}\right)}^{2}\left(\mathfrak{u}_{i}\right)-\mu_{\mathcal{G}\left(\mathfrak{h}_{j}\right)}^{2}\left(\mathfrak{u}_{i}\right)\right) \geq$ $\frac{1}{2 m n} \sum_{j=1}^{m} \sum_{i=1}^{n} 2\left(\begin{array}{c}\mu_{\mathcal{P}\left(\mathfrak{h}_{j}\right)}^{2}\left(\mathfrak{u}_{i}\right)+\nu_{\mathcal{P}\left(\mathfrak{h}_{j}\right)}^{2}\left(\mathfrak{u}_{i}\right)-\left[\mu_{\mathcal{P}\left(\mathfrak{h}_{j}\right)}^{2}\left(\mathfrak{u}_{i}\right)\right. \\ \left.+\nu_{\mathcal{P}\left(\mathfrak{h}_{j}\right)}^{2}\left(\mathfrak{u}_{i}\right)-\mu_{\mathcal{R}\left(\mathfrak{h}_{j}\right)}^{2}\left(\mathfrak{u}_{i}\right)\right]-\mu_{\mathcal{G}\left(\mathfrak{h}_{j}\right)}^{2}\left(\mathfrak{u}_{i}\right)\end{array}\right)$

$=\frac{1}{2 m n} \sum_{j=1}^{m} \sum_{i=1}^{n} 2\left(\mu_{\mathcal{R}\left(\mathfrak{h}_{j}\right)}^{2}\left(\mathfrak{u}_{i}\right)-\mu_{\mathcal{G}\left(\mathfrak{h}_{j}\right)}^{2}\left(\mathfrak{u}_{i}\right)\right) \geq 0$.

By considering all the above cases it is obtained that $\Psi_{3}((\mathcal{P}, \mathfrak{L}),(\mathcal{G}, \mathfrak{L})) \geq$ 
$\Psi_{3}((\mathcal{P}, \mathfrak{L}),(\mathcal{R}, \mathfrak{L}))$ and same way it can be proved $\Psi_{3}((\mathcal{G}, \mathfrak{L}),(\mathcal{R}, \mathfrak{L})) \geq \Psi_{3}((\mathcal{P}, \mathfrak{L}),(\mathcal{R}, \mathfrak{L}))$.

2 .

$$
\begin{gathered}
\Psi_{4}((\mathcal{P}, \mathfrak{L}),(\mathcal{G}, \mathfrak{L}))=\frac{1}{m n} \sum_{j=1}^{m} \sum_{i=1}^{n} \cos \left[\frac{\pi}{2} \max \left\{\begin{array}{l}
\left|\mu_{\mathcal{P}\left(\mathfrak{h}_{j}\right)}^{2}\left(\mathfrak{u}_{i}\right)-\mu_{\mathcal{G}\left(\mathfrak{h}_{j}\right)}^{2}\left(\mathfrak{u}_{i}\right)\right|, \\
\left.\left|\nu_{\mathcal{P}\left(\mathfrak{h}_{j}\right)}^{2}\left(\mathfrak{u}_{i}\right)-\nu_{\mathcal{G}\left(\mathfrak{h}_{j}\right)}^{2}\left(\mathfrak{u}_{i}\right)\right|\right\}
\end{array}\right]\right. \\
\Psi_{4}((\mathcal{P}, \mathfrak{L}),(\mathcal{R}, \mathfrak{L}))=\frac{1}{m n} \sum_{j=1}^{m} \sum_{i=1}^{n} \cos \left[\frac{\pi}{2} \max \left\{\begin{array}{l}
\left|\mu_{\mathcal{P}\left(\mathfrak{h}_{j}\right)}^{2}\left(\mathfrak{u}_{i}\right)-\mu_{\mathcal{R}\left(\mathfrak{h}_{j}\right)}^{2}\left(\mathfrak{u}_{i}\right)\right|, \\
\left.\left|\nu_{\mathcal{P}\left(\mathfrak{h}_{j}\right)}^{2}\left(\mathfrak{u}_{i}\right)-\nu_{\mathcal{R}\left(\mathfrak{h}_{j}\right)}^{2}\left(\mathfrak{u}_{i}\right)\right|\right\}
\end{array}\right]\right.
\end{gathered}
$$

Since $\left|\mu_{\mathcal{P}\left(\mathfrak{h}_{j}\right)}^{2}\left(\mathfrak{u}_{i}\right)-\mu_{\mathcal{G}\left(\mathfrak{h}_{j}\right)}^{2}\left(\mathfrak{u}_{i}\right)\right| \geq\left|\mu_{\mathcal{P}\left(\mathfrak{h}_{j}\right)}^{2}\left(\mathfrak{u}_{i}\right)-\mu_{\mathcal{R}\left(\mathfrak{h}_{j}\right)}^{2}\left(\mathfrak{u}_{i}\right)\right|, \mid \nu_{\mathcal{P}\left(\mathfrak{h}_{j}\right)}^{2}\left(\mathfrak{u}_{i}\right)-$ $\nu_{\mathcal{G}\left(\mathfrak{h}_{j}\right)}^{2}\left(\mathfrak{u}_{i}\right)|\geq| \nu_{\mathcal{P}\left(\mathfrak{h}_{j}\right)}^{2}\left(\mathfrak{u}_{i}\right)-\nu_{\mathcal{R}\left(\mathfrak{h}_{j}\right)}^{2}\left(\mathfrak{u}_{i}\right) \mid$ and cosine is increasing on $[0, \pi / 2]$, it is directly obtained that $\Psi_{4}((\mathcal{G}, \mathfrak{L}),(\mathcal{R}, \mathfrak{L})) \geq \Psi_{4}((\mathcal{P}, \mathfrak{L}),(\mathcal{R}, \mathfrak{L}))$.

Similarly we get $\Psi_{4}((\mathcal{G}, \mathfrak{L}),(\mathcal{R}, \mathfrak{L})) \geq \Psi_{4}((\mathcal{P}, \mathfrak{L}),(\mathcal{R}, \mathfrak{L}))$.

3.

$$
\begin{aligned}
& \Psi_{6}((\mathcal{P}, \mathfrak{L}),(\mathcal{G}, \mathfrak{L}))=1-\frac{1}{m} \sum_{j=1}^{m}\left(\begin{array}{l}
\sum_{i=1}^{n}\left|\mu_{\mathcal{P}\left(\mathfrak{h}_{j}\right)}^{2}\left(\mathfrak{u}_{i}\right)-\mu_{\mathcal{G}\left(\mathfrak{h}_{j}\right)}^{2}\left(\mathfrak{u}_{i}\right)\right| \\
+\left|\nu_{\mathcal{P}\left(\mathfrak{h}_{j}\right)}^{2}\left(\mathfrak{u}_{i}\right)-\nu_{\mathcal{G}\left(\mathfrak{h}_{j}\right)}^{2}\left(\mathfrak{u}_{i}\right)\right| \\
\sum_{i=1}^{n}\left|\mu_{\mathcal{P}\left(\mathfrak{h}_{j}\right)}^{2}\left(\mathfrak{u}_{i}\right)+\mu_{\mathcal{G}\left(\mathfrak{h}_{j}\right)}^{2}\left(\mathfrak{u}_{i}\right)\right| \\
+\left|\nu_{\mathcal{P}\left(\mathfrak{h}_{j}\right)}^{2}\left(\mathfrak{u}_{i}\right)+\nu_{\mathcal{G}\left(\mathfrak{h}_{j}\right)}^{2}\left(\mathfrak{u}_{i}\right)\right|
\end{array}\right) \\
& \Psi_{6}((\mathcal{P}, \mathfrak{L}),(\mathcal{R}, \mathfrak{L}))=1-\frac{1}{m} \sum_{j=1}^{m}\left(\begin{array}{l}
\sum_{i=1}^{n}\left|\mu_{\mathcal{P}\left(\mathfrak{h}_{j}\right)}^{2}\left(\mathfrak{u}_{i}\right)-\mu_{\mathcal{R}\left(\mathfrak{h}_{j}\right)}^{2}\left(\mathfrak{u}_{i}\right)\right| \\
+\left|\nu_{\mathcal{P}\left(\mathfrak{h}_{j}\right)}^{2}\left(\mathfrak{u}_{i}\right)-\nu_{\mathcal{G}\left(\mathfrak{h}_{j}\right)}^{2}\left(\mathfrak{u}_{i}\right)\right| \\
\sum_{i=1}^{n}\left|\mu_{\mathcal{P}\left(\mathfrak{h}_{j}\right)}^{2}\left(\mathfrak{u}_{i}\right)+\mu_{\mathcal{R}\left(\mathfrak{h}_{j}\right)}^{2}\left(\mathfrak{u}_{i}\right)\right| \\
+\left|\nu_{\mathcal{P}\left(\mathfrak{h}_{j}\right)}^{2}\left(\mathfrak{u}_{i}\right)+\nu_{\mathcal{R}\left(\mathfrak{h}_{j}\right)}^{2}\left(\mathfrak{u}_{i}\right)\right|
\end{array}\right) \\
& \text { We have, }\left(\begin{array}{l}
\left|\mu_{\mathcal{P}\left(\mathfrak{h}_{j}\right)}^{2}\left(\mathfrak{u}_{i}\right)-\mu_{\mathcal{G}\left(\mathfrak{h}_{j}\right)}^{2}\left(\mathfrak{u}_{i}\right)\right|+ \\
\left|\nu_{\mathcal{P}\left(\mathfrak{h}_{j}\right)}^{2}\left(\mathfrak{u}_{i}\right)-\nu_{\mathcal{G}\left(\mathfrak{h}_{j}\right)}^{2}\left(\mathfrak{u}_{i}\right)\right|
\end{array}\right) \leq\left(\begin{array}{l}
\left|\mu_{\mathcal{P}\left(\mathfrak{h}_{j}\right)}^{2}\left(\mathfrak{u}_{i}\right)-\mu_{\mathcal{R}\left(\mathfrak{h}_{j}\right)}^{2}\left(\mathfrak{u}_{i}\right)\right|+ \\
\left|\nu_{\mathcal{P}\left(\mathfrak{h}_{j}\right)}^{2}\left(\mathfrak{u}_{i}\right)-\nu_{\mathcal{R}\left(\mathfrak{h}_{j}\right)}^{2}\left(\mathfrak{u}_{i}\right)\right|
\end{array}\right) \\
& \Longrightarrow \frac{1}{\left(\begin{array}{l}
\left|\mu_{\mathcal{P}\left(\mathfrak{h}_{j}\right)}^{2}\left(\mathfrak{u}_{i}\right)-\mu_{\mathcal{R}\left(\mathfrak{h}_{j}\right)}^{2}\left(\mathfrak{u}_{i}\right)\right|+ \\
\left|\nu_{\mathcal{P}\left(\mathfrak{h}_{j}\right)}^{2}\left(\mathfrak{u}_{i}\right)-\nu_{\mathcal{R}\left(\mathfrak{h}_{j}\right)}^{2}\left(\mathfrak{u}_{i}\right)\right|
\end{array}\right)} \leq \frac{1}{\left(\begin{array}{l}
\left|\mu_{\mathcal{P}\left(\mathfrak{h}_{j}\right)}^{2}\left(\mathfrak{u}_{i}\right)-\mu_{\mathcal{G}\left(\mathfrak{h}_{j}\right)}^{2}\left(\mathfrak{u}_{i}\right)\right|+ \\
\left|\nu_{\mathcal{P}\left(\mathfrak{h}_{j}\right)}^{2}\left(\mathfrak{u}_{i}\right)-\nu_{\mathcal{G}\left(\mathfrak{h}_{j}\right)}^{2}\left(\mathfrak{u}_{i}\right)\right|
\end{array}\right)} \\
& \Longrightarrow 1+\frac{2\left(\mu_{\mathcal{P}\left(\mathfrak{h}_{j}\right)}^{2}\left(\mathfrak{u}_{i}\right)+\nu_{\mathcal{R}\left(\mathfrak{h}_{j}\right)}^{2}\left(\mathfrak{u}_{i}\right)\right)}{\left(\begin{array}{l}
\left|\mu_{\mathcal{P}\left(\mathfrak{h}_{j}\right)}^{2}\left(\mathfrak{u}_{i}\right)-\mu_{\mathcal{R}\left(\mathfrak{h}_{j}\right)}^{2}\left(\mathfrak{u}_{i}\right)\right|+ \\
\left|\nu_{\mathcal{P}\left(\mathfrak{h}_{j}\right)}^{2}\left(\mathfrak{u}_{i}\right)-\nu_{\mathcal{R}\left(\mathfrak{h}_{j}\right)}^{2}\left(\mathfrak{u}_{i}\right)\right|
\end{array}\right)} \leq 1+\frac{2\left(\mu_{\mathcal{P}\left(\mathfrak{h}_{j}\right)}^{2}\left(\mathfrak{u}_{i}\right)+\nu_{\mathcal{G}\left(\mathfrak{h}_{j}\right)}^{2}\left(\mathfrak{u}_{i}\right)\right)}{\left(\begin{array}{l}
\left|\mu_{\mathcal{P}\left(\mathfrak{h}_{j}\right)}^{2}\left(\mathfrak{u}_{i}\right)-\mu_{\mathcal{G}\left(\mathfrak{h}_{j}\right)}^{2}\left(\mathfrak{u}_{i}\right)\right|+ \\
\left|\nu_{\mathcal{P}\left(\mathfrak{h}_{j}\right)}^{2}\left(\mathfrak{u}_{i}\right)-\nu_{\mathcal{G}\left(\mathfrak{h}_{j}\right)}^{2}\left(\mathfrak{u}_{i}\right)\right|
\end{array}\right)}
\end{aligned}
$$




$$
\begin{aligned}
& \Longrightarrow \frac{\left(\begin{array}{l}
\left|\mu_{\mathcal{P}\left(\mathfrak{h}_{j}\right)}^{2}\left(\mathfrak{u}_{i}\right)+\mu_{\mathcal{R}\left(\mathfrak{h}_{j}\right)}^{2}\left(\mathfrak{u}_{i}\right)\right|+ \\
\left|\nu_{\mathcal{P}\left(\mathfrak{h}_{j}\right)}^{2}\left(\mathfrak{u}_{i}\right)+\nu_{\mathcal{R}\left(\mathfrak{h}_{j}\right)}^{2}\left(\mathfrak{u}_{i}\right)\right|
\end{array}\right)}{\left(\begin{array}{l}
\left|\mu_{\mathcal{P}\left(\mathfrak{h}_{j}\right)}^{2}\left(\mathfrak{u}_{i}\right)-\mu_{\mathcal{R}\left(\mathfrak{h}_{j}\right)}^{2}\left(\mathfrak{u}_{i}\right)\right|+ \\
\left|\nu_{\mathcal{P}\left(\mathfrak{h}_{j}\right)}^{2}\left(\mathfrak{u}_{i}\right)-\nu_{\mathcal{R}\left(\mathfrak{h}_{j}\right)}^{2}\left(\mathfrak{u}_{i}\right)\right|
\end{array}\right)} \leq \frac{\left(\begin{array}{l}
\left|\mu_{\mathcal{P}\left(\mathfrak{h}_{j}\right)}^{2}\left(\mathfrak{u}_{i}\right)+\mu_{\mathcal{G}\left(\mathfrak{h}_{j}\right)}^{2}\left(\mathfrak{u}_{i}\right)\right|+ \\
\left|\nu_{\mathcal{P}\left(\mathfrak{h}_{j}\right)}^{2}\left(\mathfrak{u}_{i}\right)+\nu_{\mathcal{G}\left(\mathfrak{h}_{j}\right)}^{2}\left(\mathfrak{u}_{i}\right)\right|
\end{array}\right)}{\left(\begin{array}{l}
\left|\mu_{\mathcal{P}\left(\mathfrak{h}_{j}\right)}^{2}\left(\mathfrak{u}_{i}\right)-\mu_{\mathcal{G}\left(\mathfrak{h}_{j}\right)}^{2}\left(\mathfrak{u}_{i}\right)\right|+ \\
\left|\nu_{\mathcal{P}\left(\mathfrak{h}_{j}\right)}^{2}\left(\mathfrak{u}_{i}\right)-\nu_{\mathcal{G}\left(\mathfrak{h}_{j}\right)}^{2}\left(\mathfrak{u}_{i}\right)\right|
\end{array}\right)} \\
& \Longrightarrow 1-\frac{1}{m} \sum_{j=1}^{m} \frac{\sum_{i=1}^{n}\left(\begin{array}{l}
\left|\mu_{\mathcal{P}\left(\mathfrak{h}_{j}\right)}^{2}\left(\mathfrak{u}_{i}\right)-\mu_{\mathcal{G}\left(\mathfrak{h}_{j}\right)}^{2}\left(\mathfrak{u}_{i}\right)\right|+ \\
\left|\nu_{\mathcal{P}\left(\mathfrak{h}_{j}\right)}^{2}\left(\mathfrak{u}_{i}\right)-\nu_{\mathcal{G}\left(\mathfrak{h}_{j}\right)}^{2}\left(\mathfrak{u}_{i}\right)\right|
\end{array}\right)}{\sum_{i=1}^{n}\left(\begin{array}{l}
\left|\mu_{\mathcal{P}\left(\mathfrak{h}_{j}\right)}^{2}\left(\mathfrak{u}_{i}\right)+\mu_{\mathcal{G}\left(\mathfrak{h}_{j}\right)}^{2}\left(\mathfrak{u}_{i}\right)\right|+ \\
\left|\nu_{\mathcal{P}\left(\mathfrak{h}_{j}\right)}^{2}\left(\mathfrak{u}_{i}\right)+\nu_{\mathcal{G}\left(\mathfrak{h}_{j}\right)}^{2}\left(\mathfrak{u}_{i}\right)\right|
\end{array}\right)} \geq \\
& 1-\frac{1}{m} \sum_{j=1}^{m} \frac{\sum_{i=1}^{n}\left(\begin{array}{l}
\left|\mu_{\mathcal{P}\left(\mathfrak{h}_{j}\right)}^{2}\left(\mathfrak{u}_{i}\right)-\mu_{\mathcal{R}\left(\mathfrak{h}_{j}\right)}^{2}\left(\mathfrak{u}_{i}\right)\right|+ \\
\left|\nu_{\mathcal{P}\left(\mathfrak{h}_{j}\right)}^{2}\left(\mathfrak{u}_{i}\right)-\nu_{\mathcal{R}\left(\mathfrak{h}_{j}\right)}^{2}\left(\mathfrak{u}_{i}\right)\right|
\end{array}\right)}{\sum_{i=1}^{n}\left(\begin{array}{l}
\left|\mu_{\mathcal{P}\left(\mathfrak{h}_{j}\right)}^{2}\left(\mathfrak{u}_{i}\right)+\mu_{\mathcal{R}\left(\mathfrak{h}_{j}\right)}^{2}\left(\mathfrak{u}_{i}\right)\right|+ \\
\left|\nu_{\mathcal{P}\left(\mathfrak{h}_{j}\right)}^{2}\left(\mathfrak{u}_{i}\right)+\nu_{\mathcal{R}\left(\mathfrak{h}_{j}\right)}^{2}\left(\mathfrak{u}_{i}\right)\right|
\end{array}\right)} .
\end{aligned}
$$

Thus $\Psi_{6}((\mathcal{P}, \mathfrak{L}),(\mathcal{G}, \mathfrak{L})) \geq \Psi_{6}((\mathcal{P}, \mathfrak{L}),(\mathcal{R}, \mathfrak{L}))$ and similarly it can be proven that $\Psi_{4}((\mathcal{G}, \mathfrak{L}),(\mathcal{R}, \mathfrak{L})) \geq \Psi_{4}((\mathcal{P}, \mathfrak{L}),(\mathcal{R}, \mathfrak{L}))$.

Theorem 2 For $r=1,2,3,4,5,6,8,9,10$ and $\alpha=\beta=1 / 2$,

1. $\Psi_{r}\left((\mathcal{P}, \mathfrak{L}),(\mathcal{G}, \mathfrak{L})^{c}\right)=\Psi_{r}\left((\mathcal{P}, \mathfrak{L})^{c},(\mathcal{G}, \mathfrak{L})\right)$

2. $\Psi_{r}\left((\mathcal{P}, \mathfrak{L})^{c},(\mathcal{G}, \mathfrak{L})^{c}\right)=\Psi_{r}((\mathcal{P}, \mathfrak{L}),(\mathcal{G}, \mathfrak{L}))$

3. $\Psi_{r}((\mathcal{P}, \mathfrak{L}) \sqcap(\mathcal{G}, \mathfrak{L}),(\mathcal{P}, \mathfrak{L}) \sqcup(\mathcal{G}, \mathfrak{L}))=\Psi_{r}((\mathcal{P}, \mathfrak{L}),(\mathcal{G}, \mathfrak{L})), r \neq 3,9,10$

Proof

$$
\begin{aligned}
\Psi_{1}\left((\mathcal{P}, \mathfrak{L}),(\mathcal{G}, \mathfrak{L})^{c}\right)= & \frac{\sum_{i=1}^{n} \sum_{j=1}^{m}\left(\begin{array}{l}
\min \left\{\mu_{\mathcal{P}\left(\mathfrak{h}_{j}\right)}^{2}\left(\mathfrak{u}_{i}\right), \nu_{\mathcal{G}\left(\mathfrak{h}_{j}\right)}^{2}\left(\mathfrak{u}_{i}\right)\right\}+ \\
\min \left\{\nu_{\mathcal{P}\left(\mathfrak{h}_{j}\right)}^{2}\left(\mathfrak{u}_{i}\right), \mu_{\mathcal{G}\left(\mathfrak{h}_{j}\right)}^{2}\left(\mathfrak{u}_{i}\right)\right\}
\end{array}\right)}{\sum_{i=1}^{n} \sum_{j=1}^{m}\left(\begin{array}{l}
\max \left\{\mu_{\mathcal{P}\left(\mathfrak{h}_{j}\right)}^{2}\left(\mathfrak{u}_{i}\right), \nu_{\mathcal{G}\left(\mathfrak{h}_{j}\right)}^{2}\left(\mathfrak{u}_{i}\right)\right\}+ \\
\max \left\{\nu_{\mathcal{P}\left(\mathfrak{h}_{j}\right)}^{2}\left(\mathfrak{u}_{i}\right), \mu_{\mathcal{G}\left(\mathfrak{h}_{j}\right)}^{2}\left(\mathfrak{u}_{i}\right)\right\}
\end{array}\right)} \\
= & \frac{\sum_{i=1}^{n} \sum_{j=1}^{m}\left(\begin{array}{l}
\min \left\{\nu_{\mathcal{P}\left(\mathfrak{h}_{j}\right)}^{2}\left(\mathfrak{u}_{i}\right), \mu_{\mathcal{G}\left(\mathfrak{h}_{j}\right)}^{2}\left(\mathfrak{u}_{i}\right)\right\}+ \\
\min \left\{\mu_{\mathcal{P}\left(\mathfrak{h}_{j}\right)}^{2}\left(\mathfrak{u}_{i}\right), \nu_{\mathcal{G}\left(\mathfrak{h}_{j}\right)}^{2}\left(\mathfrak{u}_{i}\right)\right\}
\end{array}\right)}{\sum_{i=1}^{n} \sum_{j=1}^{m}\left(\begin{array}{l}
\max \left\{\nu_{\mathcal{P}\left(\mathfrak{h}_{j}\right)}^{2}\left(\mathfrak{u}_{i}\right), \mu_{\mathcal{G}\left(\mathfrak{h}_{j}\right)}^{2}\left(\mathfrak{u}_{i}\right)\right\}+ \\
\max \left\{\mu_{\mathcal{P}\left(\mathfrak{h}_{j}\right)}^{2}\left(\mathfrak{u}_{i}\right), \nu_{\mathcal{G}\left(\mathfrak{h}_{j}\right)}^{2}\left(\mathfrak{u}_{i}\right)\right\}
\end{array}\right)} \\
= & \Psi_{1}\left((\mathcal{P}, \mathfrak{L})^{c},(\mathcal{G}, \mathfrak{L})\right)
\end{aligned}
$$

Similarly the rest of the proof can be done.

Theorem 3 For $r=2,3,4,5$ we get,

1. $\Psi_{r}((\mathcal{P}, \mathfrak{L}),(\mathcal{P}, \mathfrak{L}) \sqcap(\mathcal{G}, \mathfrak{L}))=\Psi_{r}((\mathcal{G}, \mathfrak{L}),(\mathcal{P}, \mathfrak{L}) \sqcup(\mathcal{G}, \mathfrak{L}))$

2. $\Psi_{r}((\mathcal{P}, \mathfrak{L}),(\mathcal{P}, \mathfrak{L}) \sqcup(\mathcal{G}, \mathfrak{L}))=\Psi_{r}((\mathcal{G}, \mathfrak{L}),(\mathcal{P}, \mathfrak{L}) \sqcap(\mathcal{G}, \mathfrak{L}))$

3. $\Psi_{r}((\mathcal{P}, \mathfrak{L}),(\mathcal{P}, \mathfrak{L})+(\mathcal{G}, \mathfrak{L}))=\Psi_{r}((\mathcal{G}, \mathfrak{L}),(\mathcal{P}, \mathfrak{L}) .(\mathcal{G}, \mathfrak{L}))$ 
4. $\Psi_{r}((\mathcal{P}, \mathfrak{L}),(\mathcal{P}, \mathfrak{L}) .(\mathcal{G}, \mathfrak{L}))=\Psi_{r}((\mathcal{G}, \mathfrak{L}),(\mathcal{P}, \mathfrak{L})+(\mathcal{G}, \mathfrak{L}))$

Proof Here we give proof for $r=3$ and the proof for remaining values of $r$ follows similarly.

$$
\begin{gathered}
\Psi_{3}((\mathcal{P}, \mathfrak{L}),(\mathcal{P}, \mathfrak{L}) \sqcap(\mathcal{G}, \mathfrak{L}))=1-\frac{1}{2 m n} \sum_{j=1}^{m} \sum_{i=1}^{n}\left(\begin{array}{l}
\left|\mu_{\mathcal{P}\left(\mathfrak{h}_{j}\right)}^{2}\left(\mathfrak{u}_{i}\right)-\mu_{\mathcal{P} \sqcap \mathcal{G}\left(\mathfrak{h}_{j}\right)}^{2}\left(\mathfrak{u}_{i}\right)\right|+ \\
\left|\nu_{\mathcal{P}\left(\mathfrak{h}_{j}\right)}^{2}\left(\mathfrak{u}_{i}\right)-\nu_{\mathcal{P} \sqcap \mathcal{G}\left(\mathfrak{h}_{j}\right)}^{2}\left(\mathfrak{u}_{i}\right)\right|+ \\
\left|\pi_{\mathcal{P}\left(\mathfrak{h}_{j}\right)}^{2}\left(\mathfrak{u}_{i}\right)-\pi_{\mathcal{P} \sqcap \mathcal{G}\left(\mathfrak{h}_{j}\right)}^{2}\left(\mathfrak{u}_{i}\right)\right|
\end{array}\right) \\
=1-\frac{1}{2 m n} \sum_{j=1}^{m} \sum_{i=1}^{n}\left(\begin{array}{l}
\left|\mu_{\mathcal{P}\left(\mathfrak{h}_{j}\right)}^{2}\left(\mathfrak{u}_{i}\right)-\min \left\{\mu_{\mathcal{P}\left(\mathfrak{h}_{j}\right)}^{2}\left(\mathfrak{u}_{i}\right), \mu_{\mathcal{G}\left(\mathfrak{h}_{j}\right)}^{2}\left(\mathfrak{u}_{i}\right)\right\}\right|+ \\
\left|\nu_{\mathcal{P}\left(\mathfrak{h}_{j}\right)}^{2}\left(\mathfrak{u}_{i}\right)-\min \left\{\nu_{\mathcal{P}\left(\mathfrak{h}_{j}\right)}^{2}\left(\mathfrak{u}_{i}\right), \nu_{\mathcal{G}\left(\mathfrak{h}_{j}\right)}^{2}\left(\mathfrak{u}_{i}\right)\right\}\right|+ \\
\left|\pi_{\mathcal{P}\left(\mathfrak{h}_{j}\right)}^{2}\left(\mathfrak{u}_{i}\right)-\min \left\{\pi_{\mathcal{P}\left(\mathfrak{h}_{j}\right)}^{2}\left(\mathfrak{u}_{i}\right), \pi_{\mathcal{G}\left(\mathfrak{h}_{j}\right)}^{2}\left(\mathfrak{u}_{i}\right)\right\}\right|
\end{array}\right) \quad(12) \\
\Psi_{3}((\mathcal{G}, \mathfrak{L}),(\mathcal{P}, \mathfrak{L}) \sqcup(\mathcal{G}, \mathfrak{L}))=1-\frac{1}{2 m n} \sum_{j=1}^{m} \sum_{i=1}^{n}\left(\begin{array}{l}
\mid \mu_{\mathcal{G}\left(\mathfrak{h}_{j}\right)}^{2}\left(\mathfrak{u}_{i}\right)-\mu_{\mathcal{P} \sqcup \mathcal{G}\left(\mathfrak{h}_{j}\right)}^{2}\left(\nu_{\mathcal{G}\left(\mathfrak{h}_{j}\right)}^{2}\left(\mathfrak{u}_{i}\right)-\nu_{\mathcal{P} \sqcup \mathcal{G}\left(\mathfrak{h}_{j}\right)}^{2}\left(\mathfrak{u}_{i}\right)|+| \pi_{\mathcal{G}\left(\mathfrak{h}_{j}\right)}^{2}\left(\mathfrak{u}_{i}\right)-\pi_{\mathcal{P} \sqcup \mathcal{G}\left(\mathfrak{h}_{j}\right)}^{2}\left(\mathfrak{u}_{i}\right) \mid\right.
\end{array}\right) \\
=1-\frac{1}{2 m n} \sum_{j=1}^{m} \sum_{i=1}^{n}\left(\begin{array}{l}
\left|\mu_{\mathcal{G}\left(\mathfrak{h}_{j}\right)}^{2}\left(\mathfrak{u}_{i}\right)-\max \left\{\mu_{\mathcal{P}\left(\mathfrak{h}_{j}\right)}^{2}\left(\mathfrak{u}_{i}\right), \mu_{\mathcal{G}\left(\mathfrak{h}_{j}\right)}^{2}\left(\mathfrak{u}_{i}\right)\right\}\right|+ \\
\left|\nu_{\mathcal{G}\left(\mathfrak{h}_{j}\right)}^{2}\left(\mathfrak{u}_{i}\right)-\max \left\{\nu_{\mathcal{P}\left(\mathfrak{h}_{j}\right)}^{2}\left(\mathfrak{u}_{i}\right), \nu_{\mathcal{G}\left(\mathfrak{h}_{j}\right)}^{2}\left(\mathfrak{u}_{i}\right)\right\}\right|+ \\
\left|\pi_{\mathcal{G}\left(\mathfrak{h}_{j}\right)}^{2}\left(\mathfrak{u}_{i}\right)-\max \left\{\pi_{\mathcal{P}\left(\mathfrak{h}_{j}\right)}^{2}\left(\mathfrak{u}_{i}\right), \pi_{\mathcal{G}\left(\mathfrak{h}_{j}\right)}^{2}\left(\mathfrak{u}_{i}\right)\right\}\right|
\end{array}\right) \quad(13)
\end{gathered}
$$

We have $\left|\pi_{\mathcal{P}\left(\mathfrak{h}_{j}\right)}^{2}\left(\mathfrak{u}_{i}\right)-\pi_{\mathcal{P} \sqcap \mathcal{G}\left(\mathfrak{h}_{j}\right)}^{2}\left(\mathfrak{u}_{i}\right)\right|=$ $\left|-\mu_{\mathcal{P}\left(\mathfrak{h}_{j}\right)}^{2}\left(\mathfrak{u}_{i}\right)-\nu_{\mathcal{P}\left(\mathfrak{h}_{j}\right)}^{2}\left(\mathfrak{u}_{i}\right)+\min \left\{\mu_{\mathcal{P}\left(\mathfrak{h}_{j}\right)}^{2}\left(\mathfrak{u}_{i}\right), \mu_{\mathcal{G}\left(\mathfrak{h}_{j}\right)}^{2}\left(\mathfrak{u}_{i}\right)\right\}+\min \left\{\nu_{\mathcal{P}\left(\mathfrak{h}_{j}\right)}^{2}\left(\mathfrak{u}_{i}\right), \nu_{\mathcal{G}\left(\mathfrak{h}_{j}\right)}^{2}\left(\mathfrak{u}_{i}\right)\right\}\right|$ and $\left|\pi_{\mathcal{G}\left(\mathfrak{h}_{j}\right)}^{2}\left(\mathfrak{u}_{i}\right)-\pi_{\mathcal{P} \sqcup \mathcal{G}\left(\mathfrak{h}_{j}\right)}^{2}\left(\mathfrak{u}_{i}\right)\right|=$ $\left|-\mu_{\mathcal{G}\left(\mathfrak{h}_{j}\right)}^{2}\left(\mathfrak{u}_{i}\right)-\nu_{\mathcal{G}\left(\mathfrak{h}_{j}\right)}^{2}\left(\mathfrak{u}_{i}\right)+\max \left\{\mu_{\mathcal{P}\left(\mathfrak{h}_{j}\right)}^{2}\left(\mathfrak{u}_{i}\right), \mu_{\mathcal{G}\left(\mathfrak{h}_{j}\right)}^{2}\left(\mathfrak{u}_{i}\right)\right\}+\min \left\{\nu_{\mathcal{P}\left(\mathfrak{h}_{j}\right)}^{2}\left(\mathfrak{u}_{i}\right), \nu_{\mathcal{G}\left(\mathfrak{h}_{j}\right)}^{2}\left(\mathfrak{u}_{i}\right)\right\}\right|$.

Consider all the four cases;

Case 1: If $\mu_{\mathcal{P}\left(\mathfrak{h}_{j}\right)}^{2}\left(\mathfrak{u}_{i}\right) \leq \mu_{\mathcal{G}\left(\mathfrak{h}_{j}\right)}^{2}\left(\mathfrak{u}_{i}\right)$ and $\nu_{\mathcal{P}\left(\mathfrak{h}_{j}\right)}^{2}\left(\mathfrak{u}_{i}\right) \leq \nu_{\mathcal{G}\left(\mathfrak{h}_{j}\right)}^{2}\left(\mathfrak{u}_{i}\right)$, then $\left|\pi_{\mathcal{P}\left(\mathfrak{h}_{j}\right)}^{2}\left(\mathfrak{u}_{i}\right)-\pi_{\mathcal{P} \sqcap \mathcal{G}\left(\mathfrak{h}_{j}\right)}^{2}\left(\mathfrak{u}_{i}\right)\right|$

$$
\begin{aligned}
& =\left|-\mu_{\mathcal{P}\left(\mathfrak{h}_{j}\right)}^{2}\left(\mathfrak{u}_{i}\right)-\nu_{\mathcal{P}\left(\mathfrak{h}_{j}\right)}^{2}\left(\mathfrak{u}_{i}\right)+\mu_{\mathcal{P}\left(\mathfrak{h}_{j}\right)}^{2}\left(\mathfrak{u}_{i}\right)+\nu_{\mathcal{G}\left(\mathfrak{h}_{j}\right)}^{2}\left(\mathfrak{u}_{i}\right)\right| \\
& =\left|\nu_{\mathcal{G}\left(\mathfrak{h}_{j}\right)}^{2}\left(\mathfrak{u}_{i}\right)-\nu_{\mathcal{P}\left(\mathfrak{h}_{j}\right)}^{2}\left(\mathfrak{u}_{i}\right)\right| \\
\mid \pi_{\mathcal{G}\left(\mathfrak{h}_{j}\right)}^{2}\left(\mathfrak{u}_{i}\right)- & \pi_{\mathcal{P} \sqcup \mathcal{G}\left(\mathfrak{h}_{j}\right)}^{2}\left(\mathfrak{u}_{i}\right) \mid \\
& =\left|-\mu_{\mathcal{G}\left(\mathfrak{h}_{j}\right)}^{2}\left(\mathfrak{u}_{i}\right)-\nu_{\mathcal{G}\left(\mathfrak{h}_{j}\right)}^{2}\left(\mathfrak{u}_{i}\right)+\mu_{\mathcal{G}\left(\mathfrak{h}_{j}\right)}^{2}\left(\mathfrak{u}_{i}\right)+\nu_{\mathcal{P}\left(\mathfrak{h}_{j}\right)}^{2}\left(\mathfrak{u}_{i}\right)\right| \\
& =\left|\nu_{\mathcal{P}\left(\mathfrak{h}_{j}\right)}^{2}\left(\mathfrak{u}_{i}\right)-\nu_{\mathcal{G}\left(\mathfrak{h}_{j}\right)}^{2}\left(\mathfrak{u}_{i}\right)\right| .
\end{aligned}
$$

Case 2: If $\mu_{\mathcal{P}\left(\mathfrak{h}_{j}\right)}^{2}\left(\mathfrak{u}_{i}\right) \leq \mu_{\mathcal{G}\left(\mathfrak{h}_{j}\right)}^{2}\left(\mathfrak{u}_{i}\right)$ and $\nu_{\mathcal{G}\left(\mathfrak{h}_{j}\right)}^{2}\left(\mathfrak{u}_{i}\right) \leq \nu_{\mathcal{P}\left(\mathfrak{h}_{j}\right)}^{2}\left(\mathfrak{u}_{i}\right)$, then

$$
\begin{aligned}
\left|\pi_{\mathcal{P}\left(\mathfrak{h}_{j}\right)}^{2}\left(\mathfrak{u}_{i}\right)-\pi_{\mathcal{P} \sqcap \mathcal{G}\left(\mathfrak{h}_{j}\right)}^{2}\left(\mathfrak{u}_{i}\right)\right| & \\
= & \left|-\mu_{\mathcal{P}\left(\mathfrak{h}_{j}\right)}^{2}\left(\mathfrak{u}_{i}\right)-\nu_{\mathcal{P}\left(\mathfrak{h}_{j}\right)}^{2}\left(\mathfrak{u}_{i}\right)+\mu_{\mathcal{P}\left(\mathfrak{h}_{j}\right)}^{2}\left(\mathfrak{u}_{i}\right)+\nu_{\mathcal{G}\left(\mathfrak{h}_{j}\right)}^{2}\left(\mathfrak{u}_{i}\right)\right| \\
& =\left|\nu_{\mathcal{G}\left(\mathfrak{h}_{j}\right)}^{2}\left(\mathfrak{u}_{i}\right)-\nu_{\mathcal{P}\left(\mathfrak{h}_{j}\right)}^{2}\left(\mathfrak{u}_{i}\right)\right|
\end{aligned}
$$




$$
\begin{aligned}
\mid \pi_{\mathcal{G}\left(\mathfrak{h}_{j}\right)}^{2}\left(\mathfrak{u}_{i}\right) & -\pi_{\mathcal{P} \sqcup \mathcal{G}\left(\mathfrak{h}_{j}\right)}^{2}\left(\mathfrak{u}_{i}\right) \mid \\
= & \left|-\mu_{\mathcal{G}\left(\mathfrak{h}_{j}\right)}^{2}\left(\mathfrak{u}_{i}\right)-\nu_{\mathcal{G}\left(\mathfrak{h}_{j}\right)}^{2}\left(\mathfrak{u}_{i}\right)+\nu_{\mathcal{G}\left(\mathfrak{h}_{j}\right)}^{2}\left(\mathfrak{u}_{i}\right)+\nu_{\mathcal{P}\left(\mathfrak{h}_{j}\right)}^{2}\left(\mathfrak{u}_{i}\right)\right| \\
= & \left|\nu_{\mathcal{P}\left(\mathfrak{h}_{j}\right)}^{2}\left(\mathfrak{u}_{i}\right)-\nu_{\mathcal{G}\left(\mathfrak{h}_{j}\right)}^{2}\left(\mathfrak{u}_{i}\right)\right| .
\end{aligned}
$$

Case 3: If $\mu_{\mathcal{G}\left(\mathfrak{h}_{j}\right)}^{2}\left(\mathfrak{u}_{i}\right) \leq \mu_{\mathcal{P}\left(\mathfrak{h}_{j}\right)}^{2}\left(\mathfrak{u}_{i}\right)$ and $\nu_{\mathcal{G}\left(\mathfrak{h}_{j}\right)}^{2}\left(\mathfrak{u}_{i}\right) \leq \nu_{\mathcal{P}\left(\mathfrak{h}_{j}\right)}^{2}\left(\mathfrak{u}_{i}\right)$, then

$$
\begin{aligned}
\mid \pi_{\mathcal{P}\left(\mathfrak{h}_{j}\right)}^{2}\left(\mathfrak{u}_{i}\right) & -\pi_{\mathcal{P} \sqcap \mathcal{G}\left(\mathfrak{h}_{j}\right)}^{2}\left(\mathfrak{u}_{i}\right) \mid \\
= & \left|-\mu_{\mathcal{P}\left(\mathfrak{h}_{j}\right)}^{2}\left(\mathfrak{u}_{i}\right)-\nu_{\mathcal{P}\left(\mathfrak{h}_{j}\right)}^{2}\left(\mathfrak{u}_{i}\right)+\mu_{\mathcal{G}\left(\mathfrak{h}_{j}\right)}^{2}\left(\mathfrak{u}_{i}\right)+\nu_{\mathcal{P}\left(\mathfrak{h}_{j}\right)}^{2}\left(\mathfrak{u}_{i}\right)\right| \\
= & \left|\mu_{\mathcal{G}\left(\mathfrak{h}_{j}\right)}^{2}\left(\mathfrak{u}_{i}\right)-\mu_{\mathcal{P}\left(\mathfrak{h}_{j}\right)}^{2}\left(\mathfrak{u}_{i}\right)\right| \\
\mid \pi_{\mathcal{G}\left(\mathfrak{h}_{j}\right)}^{2}\left(\mathfrak{u}_{i}\right) & -\pi_{\mathcal{P} \sqcup \mathcal{G}\left(\mathfrak{h}_{j}\right)}^{2}\left(\mathfrak{u}_{i}\right) \mid \\
= & \left|-\mu_{\mathcal{G}\left(\mathfrak{h}_{j}\right)}^{2}\left(\mathfrak{u}_{i}\right)-\nu_{\mathcal{G}\left(\mathfrak{h}_{j}\right)}^{2}\left(\mathfrak{u}_{i}\right)+\mu_{\mathcal{P}\left(\mathfrak{h}_{j}\right)}^{2}\left(\mathfrak{u}_{i}\right)+\nu_{\mathcal{G}\left(\mathfrak{h}_{j}\right)}^{2}\left(\mathfrak{u}_{i}\right)\right| \\
= & \left|\mu_{\mathcal{P}\left(\mathfrak{h}_{j}\right)}^{2}\left(\mathfrak{u}_{i}\right)-\mu_{\mathcal{G}\left(\mathfrak{h}_{j}\right)}^{2}\left(\mathfrak{u}_{i}\right)\right| .
\end{aligned}
$$

Case 4: If $\mu_{\mathcal{G}\left(\mathfrak{h}_{j}\right)}^{2}\left(\mathfrak{u}_{i}\right) \leq \mu_{\mathcal{P}\left(\mathfrak{h}_{j}\right)}^{2}\left(\mathfrak{u}_{i}\right)$ and $\nu_{\mathcal{P}\left(\mathfrak{h}_{j}\right)}^{2}\left(\mathfrak{u}_{i}\right) \leq \nu_{\mathcal{G}\left(\mathfrak{h}_{j}\right)}^{2}\left(\mathfrak{u}_{i}\right)$, then

$$
\begin{aligned}
\mid \pi_{\mathcal{P}\left(\mathfrak{h}_{j}\right)}^{2}\left(\mathfrak{u}_{i}\right) & -\pi_{\mathcal{P} \sqcap \mathcal{G}\left(\mathfrak{h}_{j}\right)}^{2}\left(\mathfrak{u}_{i}\right) \mid \\
= & \left|-\mu_{\mathcal{P}\left(\mathfrak{h}_{j}\right)}^{2}\left(\mathfrak{u}_{i}\right)-\nu_{\mathcal{P}\left(\mathfrak{h}_{j}\right)}^{2}\left(\mathfrak{u}_{i}\right)+\mu_{\mathcal{G}\left(\mathfrak{h}_{j}\right)}^{2}\left(\mathfrak{u}_{i}\right)+\nu_{\mathcal{G}\left(\mathfrak{h}_{j}\right)}^{2}\left(\mathfrak{u}_{i}\right)\right| \\
= & \left|\mu_{\mathcal{G}\left(\mathfrak{h}_{j}\right)}^{2}\left(\mathfrak{u}_{i}\right)-\mu_{\mathcal{P}\left(\mathfrak{h}_{j}\right)}^{2}\left(\mathfrak{u}_{i}\right)+\nu_{\mathcal{G}\left(\mathfrak{h}_{j}\right)}^{2}\left(\mathfrak{u}_{i}\right)-\nu_{\mathcal{P}\left(\mathfrak{h}_{j}\right)}^{2}\left(\mathfrak{u}_{i}\right)\right| \\
\mid \pi_{\mathcal{G}\left(\mathfrak{h}_{j}\right)}^{2}\left(\mathfrak{u}_{i}\right) & -\pi_{\mathcal{P} \sqcup \mathcal{G}\left(\mathfrak{h}_{j}\right)}^{2}\left(\mathfrak{u}_{i}\right) \mid \\
= & \left|-\mu_{\mathcal{G}\left(\mathfrak{h}_{j}\right)}^{2}\left(\mathfrak{u}_{i}\right)-\nu_{\mathcal{G}\left(\mathfrak{h}_{j}\right)}^{2}\left(\mathfrak{u}_{i}\right)+\mu_{\mathcal{P}\left(\mathfrak{h}_{j}\right)}^{2}\left(\mathfrak{u}_{i}\right)+\nu_{\mathcal{G}\left(\mathfrak{h}_{j}\right)}^{2}\left(\mathfrak{u}_{i}\right)\right| \\
= & \left|\mu_{\mathcal{P}\left(\mathfrak{h}_{j}\right)}^{2}\left(\mathfrak{u}_{i}\right)-\mu_{\mathcal{G}\left(\mathfrak{h}_{j}\right)}^{2}\left(\mathfrak{u}_{i}\right)+\nu_{\mathcal{P}\left(\mathfrak{h}_{j}\right)}^{2}\left(\mathfrak{u}_{i}\right)-\nu_{\mathcal{G}\left(\mathfrak{h}_{j}\right)\left(\mathfrak{u}_{i}\right)}\right| .
\end{aligned}
$$

In all the four cases we have

$$
\left|\pi_{\mathcal{P}\left(\mathfrak{h}_{j}\right)}^{2}\left(\mathfrak{u}_{i}\right)-\pi_{\mathcal{P} \sqcap \mathcal{G}\left(\mathfrak{h}_{j}\right)}^{2}\left(\mathfrak{u}_{i}\right)\right|=\left|\pi_{\mathcal{G}\left(\mathfrak{h}_{j}\right)}^{2}\left(\mathfrak{u}_{i}\right)-\pi_{\mathcal{P} \sqcup \mathcal{G}\left(\mathfrak{h}_{j}\right)}^{2}\left(\mathfrak{u}_{i}\right)\right| .
$$

Thus,

$$
\Psi_{3}((\mathcal{P}, \mathfrak{L}),(\mathcal{P}, \mathfrak{L}) \sqcap(\mathcal{G}, \mathfrak{L}))=\Psi_{3}((\mathcal{G}, \mathfrak{L}),(\mathcal{P}, \mathfrak{L}) \sqcup(\mathcal{G}, \mathfrak{L})) .
$$

Similarly, the remaining properties can be done.

\section{Applications of Similarity Measures of PFSS}

Here, a comparison study is carried out and that shows the superiority of the measure that we introduced. The applications of the obtained results are explained through the method of cluster analysis. A suitable clustering algorithm is proposed and displayed an example. 


\subsection{Comparative Study}

This section marks out the advantages of similarity measures of PFSS over the existing measures. Table 1 contains already established similarity measures for IFSS.

Table 1 Table showing already established similarity measures

\begin{tabular}{|c|c|}
\hline Authors & $\begin{array}{l}\text { Similarity measures } \\
\Psi((\mathcal{P}, \mathfrak{L}),(\mathcal{G}, \mathfrak{L}))\end{array}$ \\
\hline Çağman et al. [7] & 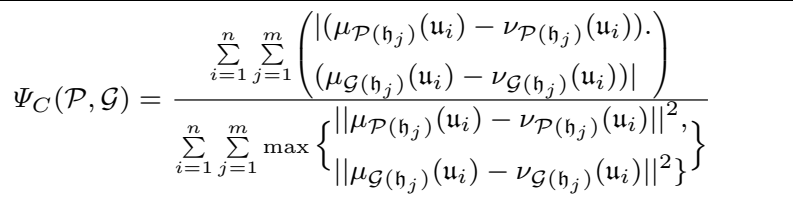 \\
\hline Muthukumar et al. [24] & $\Psi_{M}(\mathcal{P}, \mathcal{G})=\frac{\sum_{j=1}^{m}\left(\begin{array}{l}\mu_{\mathcal{P}\left(\mathfrak{h}_{j}\right)}(\mathfrak{u}) \cdot \mu_{\mathcal{G}\left(\mathfrak{h}_{j}\right)}(\mathfrak{u})+ \\
\nu_{\mathcal{P}\left(\mathfrak{h}_{j}\right)}(\mathfrak{u}) \cdot \nu_{\mathcal{G}\left(\mathfrak{h}_{j}\right)}(\mathfrak{u})\end{array}\right)}{\sum_{j=1}^{m}\left(\begin{array}{l}\left.\left(\mu_{\mathcal{P}\left(\mathfrak{h}_{j}\right)}(\mathfrak{u})\right)^{2} \vee \mu_{\mathcal{G}\left(\mathfrak{h}_{j}\right)}(\mathfrak{u})\right)^{2}+ \\
\left.\left(\nu_{\mathcal{P}\left(\mathfrak{h}_{j}\right)}(\mathfrak{u})\right)^{2} \vee \nu_{\mathcal{G}\left(\mathfrak{h}_{j}\right)}(\mathfrak{u})\right)^{2}\end{array}\right)}$ \\
\hline Mukherjee et al. [23] & $\Psi_{M u}(\mathcal{P}, \mathcal{G})=\frac{1}{1+\frac{1}{2 m n} \sum_{i=1}^{n} \sum_{j=1}^{m}\left(\begin{array}{l}\left|\mu_{\mathcal{P}\left(\mathfrak{h}_{j}\right)}\left(\mathfrak{u}_{i}\right)-\mu_{\mathcal{G}\left(\mathfrak{h}_{j}\right)}\left(\mathfrak{u}_{i}\right)\right|^{2}+ \\
\left|\nu_{\mathcal{P}\left(\mathfrak{h}_{j}\right)}\left(\mathfrak{u}_{i}\right)-\nu_{\mathcal{G}\left(\mathfrak{h}_{j}\right)}\left(\mathfrak{u}_{i}\right)\right|^{2}\end{array}\right)^{\frac{1}{2}}}$ \\
\hline Sarala et al. [29] & $\Psi_{S}(\mathcal{P}, \mathcal{G})=1-\frac{1}{2 m n} \sum_{i=1}^{n} \sum_{j=1}^{m}\left(\begin{array}{l}\left|\mu_{\mathcal{P}\left(\mathfrak{h}_{j}\right)}\left(\mathfrak{u}_{i}\right)-\mu_{\mathcal{G}\left(\mathfrak{h}_{j}\right)}\left(\mathfrak{u}_{i}\right)\right|+ \\
\left|\nu_{\mathcal{P}\left(\mathfrak{h}_{j}\right)}\left(\mathfrak{u}_{i}\right)-\nu_{\mathcal{G}\left(\mathfrak{h}_{j}\right)}\left(\mathfrak{u}_{i}\right)\right|\end{array}\right)$ \\
\hline
\end{tabular}

A similarity measure for IFSS cannot be directly adopted to a similarity measure for PFSS, even though PFSS is a generalization of IFSS. For example, consider the similarity measure $S_{C}(\mathcal{P}, \mathcal{G})$ given in the Table 1 . The following example shows that $\Psi_{C}(\mathcal{P}, \mathcal{G})=0$ for entirely different PFSS $\mathcal{P}$ and $\mathcal{G}$.

Example 1 Consider the universal set $\mho=\left\{u_{1}, u_{2}\right\}$ and the parameter set $\mathfrak{L}=\left\{e_{1}, e_{2}\right\}$. The PFSSs $\left(\mathcal{P}_{1}, \mathfrak{L}\right)$ and $\left(\mathcal{P}_{2}, \mathfrak{L}\right)$ is given as,

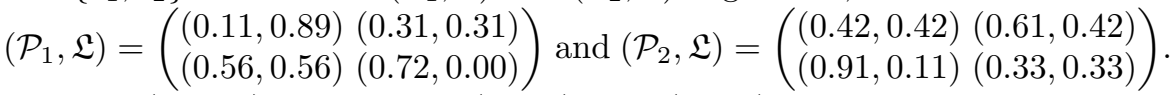
Then $S_{C}\left(\mathcal{P}_{1}, \mathcal{P}_{2}\right)=0$ where $\left(\mathcal{P}_{1}, \mathfrak{L}\right)$ and $\left(\mathcal{P}_{2}, \mathfrak{L}\right)$ are two different PFSSs. Therefore $\Psi_{C}(\mathcal{P}, \mathcal{G})$ is not a similarity measure for PFSSs. Note that the proposed similarity measure, for example $\Psi_{3}\left(\mathcal{P}_{1}, \mathcal{P}_{2}\right)$ of $\left(\mathcal{P}_{1}, \mathfrak{L}\right)$ and $\left(\mathcal{P}_{2}, \mathfrak{L}\right)$ is 0.53 .

The next example describes a comparative analysis of the new notion of similarity measure with the remaining notions of similarity measures within Table 1 in the context of a pattern recognition problem. 
Example 2 Take three patterns $\mathfrak{T}_{1}, \mathfrak{T}_{2}, \mathfrak{T}_{3}$ and an unknown pattern $\mathfrak{U}$ into account. It is to be identified that the pattern $\mathfrak{Q}$ belongs to which class i.e., either $\mathfrak{T}_{1}, \mathfrak{T}_{2}$ or $\mathfrak{T}_{3}$. The description of each patterns in terms of PFSS is given below.

Consider the universal set $\mho=\left\{u_{1}, u_{2}\right\}$ and the parameter set $\mathfrak{L}=\left\{e_{1}, e_{2}\right\}$. Then the patterns are given as,

$$
\begin{aligned}
& \mathfrak{T}_{1}=\left(\begin{array}{ll}
(0.19,0.31) & (0.60,0.12) \\
(0.58,0.22) & (0.79,0.11)
\end{array}\right), \mathfrak{T}_{2}=\left(\begin{array}{cc}
(0.25,0.25) & (0.26,0.30) \\
(0.61,0.22) & (0.61,0.31)
\end{array}\right), \\
& \mathfrak{T}_{3}=\left(\begin{array}{ll}
(0.34,0.64) & (0.59,0.15) \\
(0.29,0.12) & (0.49,0.18)
\end{array}\right), \mathfrak{U}=\left(\begin{array}{ll}
(0.28,0.48) & (0.41,0.19) \\
(0.44,0.16) & (0.66,0.23)
\end{array}\right) .
\end{aligned}
$$

The table 2 explains a situation in which pattern recognition using existing similarity measures fail.

Table 2 Pattern recognition

\begin{tabular}{lllll} 
& $\Psi\left(\mathfrak{T}_{1}, \mathfrak{U}\right)$ & $\Psi\left(\mathfrak{T}_{2}, \mathfrak{U}\right)$ & $\Psi\left(\mathfrak{T}_{3}, \mathfrak{U}\right)$ & Decision \\
\hline$\Psi_{M}$ & $\mathbf{0 . 7 4}$ & $\mathbf{0 . 7 4}$ & $\mathbf{0 . 7 4}$ & Can not be classified \\
$\Psi_{M u}$ & $\mathbf{0 . 9 6}$ & $\mathbf{0 . 9 6}$ & $\mathbf{0 . 9 6}$ & Can not be classified \\
$\Psi_{S}$ & 0.88 & $\mathbf{0 . 8 9}$ & $\mathbf{0 . 8 9}$ & Can not be classified \\
$\Psi_{3}$ (Proposed) & 0.82 & 0.86 & 0.82 & $\mathfrak{P}_{2}$ \\
\hline
\end{tabular}

\subsection{PFSSs Clustering Algorithm}

Clustering analysis has been extensively studied and applied in heterogeneous fields. A straight forward and practical algorithm is proposed in the PFSSs environment. Before doing this some useful definitions are incorporated.

Definition 11 Let $\left(\mathcal{P}_{k}, \mathfrak{L}\right)$ be $k$ number of PFSSs over $\mho \mathcal{M}=\left[M_{u v}\right]_{k \times k}$ is called similarity matrix if $\mathcal{M}_{u v}=\Psi\left(\left(\mathcal{P}_{u}, \mathfrak{L}\right),\left(\mathcal{P}_{v}, \mathfrak{L}\right)\right)$ and $\Psi\left(\left(\mathcal{P}_{u}, \mathfrak{L}\right),\left(\mathcal{P}_{v}, \mathfrak{L}\right)\right)$ denotes the similarity measure between $\left(\mathcal{P}_{u}, \mathfrak{L}\right)$ and $\left(\mathcal{P}_{v}, \mathfrak{L}\right)$ which satisfying,

$$
\begin{aligned}
\text { i } 0 \leq \mathcal{M}_{u v} \leq 1, u, v=1,2, \cdots, k \\
\text { ii } \mathcal{M}_{u u}=1, u=1,2, \cdots, k \\
\text { iii } \mathcal{M}_{u v}=\mathcal{M}_{v u}, u, v=1,2, \cdots, k
\end{aligned}
$$

Definition 12 [32] Let $\mathcal{M}=\left[\mathcal{M}_{u v}\right]_{k \times k}$ be a similarity matrix, if $\mathcal{M}^{2}=$ $\mathcal{M} \circ \mathcal{M}=\left[\mathcal{M}_{u v}\right]_{k} \times k$; then $\mathcal{M}^{2}$ is defined as composition matrix of $\mathcal{M}$, where $\mathcal{M}_{u v}=\max _{p}\left\{\min \left\{\mathcal{M}_{u p}, \mathcal{M}_{p v}\right\}\right\}, u, v=1,2, \cdots, m$.

Definition $13[32]$ Let $\mathcal{M}=\left(\mathcal{M}_{u v}\right)_{k \times k}$ be a similarity matrix. Then in accordance with a finite compositions $\mathcal{M} \rightarrow \mathcal{M}^{2} \rightarrow \mathcal{M}^{4} \rightarrow, \cdots, \mathcal{M}^{2 p} \rightarrow \cdots$, there must exist an integer $p>0$ such that $\mathcal{M}^{2 p}=\mathcal{M}^{2(p+1)}$, and $\mathcal{M}^{2 p}$ is known as equivalent similarity matrix. 
Definition 14 [32] For a given equivalent similarity matrix $\mathcal{M}=\left(\mathcal{M}_{u v}\right)_{k \times k}$, $\Gamma$-cutting matrix of $\mathcal{M}$ is $\mathcal{M}^{\Gamma}=\left[\mathcal{M}_{u v}^{\Gamma}\right]_{k \times k}$ where,

$$
\mathcal{M}_{u v}^{\Gamma}=\left\{\begin{array}{ll}
1 & \text { if } \quad \mathcal{M}_{u v} \geq \Gamma \\
0 & \text { if } \quad \mathcal{M}_{u v}<\Gamma
\end{array} .\right.
$$

and $\Gamma \in[0,1]$ is the confidence interval.

Suppose we have $k$ alternatives $P_{1}, P_{2}, \cdots, P_{k}$ characterised by $n$ attributes, $a_{1}, a_{2}, \cdots, a_{n}$. Further, consider there are $r$ experts $\mathfrak{h}_{1}, \mathfrak{h}_{2}, \cdots, \mathfrak{h}_{r}$ where each of them provide their preferences in terms of PFSSs in accordance with attributes given. Our goal is to group the given alternatives $P_{u}(u=1,2, \cdots, k)$ by considering the opinions of each experts with equal importance. The step by step algorithm for fulfilling our goal is given below.

Step 1: Represent experts' view points about each alternatives with respect the attributes in terms of PFSSs. Here, $\left\{a_{1}, a_{2}, \cdots a_{n}\right\}$ is the universal set and $\left\{\mathfrak{h}_{1}, \mathfrak{h}_{2}, \cdots, \mathfrak{h}_{r}\right\}$ is parameter set.

Step 2: Construct the similarity matrix $\mathcal{M}=\left(m_{u v}\right)_{k \times k}$, where $m_{u v}=$ $S_{3}\left(P_{u}, P_{v}\right)$ and it can be rewritten as,

$$
\Psi_{3}((\mathcal{P}, \mathfrak{L}),(\mathcal{G}, \mathfrak{L}))=1-\frac{1}{2 m n} \sum_{j=1}^{m} \sum_{i=1}^{n}\left(\begin{array}{l}
\left|\mu_{\mathcal{P}\left(\mathfrak{h}_{j}\right)}^{2}\left(\mathfrak{u}_{i}\right)-\mu_{\mathcal{G}\left(\mathfrak{h}_{j}\right)}^{2}\left(\mathfrak{u}_{i}\right)\right|+ \\
\left|\nu_{\mathcal{P}\left(\mathfrak{h}_{j}\right)}^{2}\left(\mathfrak{u}_{i}\right)-\nu_{\mathcal{G}\left(\mathfrak{h}_{j}\right)}^{2}\left(\mathfrak{u}_{i}\right)\right|+ \\
\left|\pi_{\mathcal{P}\left(\mathfrak{h}_{j}\right)}^{2}\left(\mathfrak{u}_{i}\right)-\pi_{\mathcal{G}\left(\mathfrak{h}_{j}\right)}^{2}\left(\mathfrak{u}_{i}\right)\right|
\end{array}\right)
$$

Step 3: Construct the corresponding similarity matrix $\mathcal{M}^{2 k}$, where $k=1,2,3, \ldots$ such as $\mathcal{M} \rightarrow \mathcal{M}^{2} \rightarrow \mathcal{M}^{4} \rightarrow, \cdots, \mathcal{M}^{2 k} \rightarrow \cdots$, until $\mathcal{M}^{2 k}=\mathcal{M}^{2 k+1}$.

Step 4: Using definition 14, formulate $\Gamma$-cutting matrix $\mathcal{M}^{\Gamma}=$ $\left[\mathcal{M}_{u v}^{\Gamma}\right]_{k \times k}$ for a confidence interval $\Gamma$.

Step 5: Classify the PFSSs according to the rule; If every elements in the $u^{t h}$ row (column) of $\mathcal{M}^{\Gamma}$ matrix are identical as the corresponding elements in $v^{\text {th }}$ row (column) of $\mathcal{M}^{\Gamma}$ matrix then respective $P_{u}$ s belong to the same class.

\subsubsection{Illustrative Example}

Consider the situation explained by Garg et al. in the paper [11] with the following additional aspect. Instead of considering the opinion of a single expert, consider five experts and represent each of their explanations separately. The PFSSs are represented for the ten software evaluated by five experts concerning the four criteria skill in the image processing (IP), measurement equipment for coordinate/distance/area/volume (ME), producing contour lines by applying digital elevation models (DEM)/digital surface models (DEM/DSM) and, 
production of 3D modeling/texturing abilities (PM/TA) which are given in tables $3,4, \cdots, 12$.

Table 3 Table showing explanations of software $\Delta_{1}$

\begin{tabular}{lllll} 
& IP & ME & DEM/DSM & PM/TA \\
& & & & \\
Expert $_{1}$ & $(0.481,0.392)$ & $(0.690,0.211)$ & $(0.772,0.233)$ & $(0.781,0.240)$ \\
Expert $_{2}$ & $(0.890,0.200)$ & $(0.672,0.312)$ & $(0.691,0.481)$ & $(0.611,0.352)$ \\
Expert $_{3}$ & $(0.783,0.152)$ & $(0.662,0.282)$ & $(0.672,0.411)$ & $(0.920,0.290)$ \\
Expert $_{4}$ & $(0.872,0.272)$ & $(0.422,0.591)$ & $(0.823,0.513)$ & $(0.740,0.060)$ \\
Expert $_{5}$ & $(0.513,0.581)$ & $(0.661,0.041)$ & $(0.691,0.231)$ & $(0.760,0.380)$ \\
\hline
\end{tabular}

Table 4 Table showing explanations of software $\Delta_{2}$

\begin{tabular}{lllll}
\hline & IP & ME & DEM/DSM & PM/TA \\
& & & & \\
Expert $_{1}$ & $(0.586,0.318)$ & $(0.662,0.332)$ & $(0.471,0.481)$ & $(0.371,0.542)$ \\
Expert $_{2}$ & $(0.890,0.140)$ & $(0.910,0.290)$ & $(0.760,0.550)$ & $(0.640,0.530)$ \\
Expert $_{3}$ & $(0.881,0.172)$ & $(0.702,0.261)$ & $(0.661,0.321)$ & $(0.873,0.143)$ \\
Expert $_{4}$ & $(0.770,0.450)$ & $(0.271,0.682)$ & $(0.721,0.561)$ & $(0.822,0.292)$ \\
Expert $_{5}$ & $(0.572,0.573)$ & $(0.773,0.133)$ & $(0.741,0.234)$ & $(0.774,0.264)$
\end{tabular}

Table 5 Table showing explanations of software $\Delta_{3}$

\begin{tabular}{lllll} 
& IP & ME & DEM/DSM & PM/TA \\
& & & & \\
Expert $_{1}$ & $(0.251,0.451)$ & $(0.512,0.321)$ & $(0.322,0.543)$ & $(0.450,0.331)$ \\
Expert $_{2}$ & $(0.890,0.011)$ & $(0.641,0.442)$ & $(0.724,0.342)$ & $(0.452,0.442)$ \\
Expert $_{3}$ & $(0.754,0.254)$ & $(0.781,0.222)$ & $(0.782,0.561)$ & $(0.889,0.332)$ \\
Expert $_{4}$ & $(0.778,0.144)$ & $(0.343,0.853)$ & $(0.872,0.591)$ & $(0.521,0.311)$ \\
Expert $_{5}$ & $(0.471,0.672)$ & $(0.442,0.031)$ & $(0.713,0.343)$ & $(0.783,0.371)$ \\
\hline
\end{tabular}

Table 6 Table showing explanations of software $\Delta_{4}$

$\begin{array}{lllll} & \text { IP } & \text { ME } & \text { DEM/DSM } & \text { PM/TA } \\ & & & & \\ \text { Expert }_{1} & (0.342,0.272) & (0.551,0.241) & (0.563,0.233) & (0.713,0.291) \\ \text { Expert }_{2} & (0.881,0.000) & (0.570,0.360) & (0.730,0.450) & (0.880,0.441) \\ \text { Expert }_{3} & (0.811,0.156) & (0.783,0.223) & (0.891,0.452) & (0.885,0.465) \\ \text { Expert }_{4} & (0.571,0.572) & (0.633,0.173) & (0.771,0.441) & (0.662,0.233) \\ \text { Expert }_{5} & (0.554,0.234) & (0.714,0.312) & (0.892,0.342) & (0.662,0.142)\end{array}$


Table 7 Table showing explanations of software $\Delta_{5}$

$\begin{array}{lllll} & \text { IP } & \text { ME } & \text { DEM/DSM } & \text { PM/TA } \\ & & & & \\ \text { Expert }_{1} & (0.761,0.331) & (0.322,0.682) & (0.551,0.651) & (0.273,0.673) \\ \text { Expert }_{2} & (0.783,0.191) & (0.681,0.352) & (0.666,0.546) & (0.462,0.600) \\ \text { Expert }_{3} & (0.680,0.272) & (0.750,0.262) & (0.752,0.431) & (0.891,0.289) \\ \text { Expert }_{4} & (0.560,0.150) & (0.570,0.271) & (0.781,0.654) & (0.554,0.252) \\ \text { Expert }_{5} & (0.514,0.592) & (0.622,0.562) & (0.721,0.322) & (0.773,0.363)\end{array}$

Table 8 Table showing explanations of software $\Delta_{6}$

\begin{tabular}{lllll} 
& IP & ME & DEM/DSM & PM/TA \\
& & & & \\
Expert $_{1}$ & $(0.721,0.321)$ & $(0.445,0.274)$ & $(0.672,0.322)$ & $(0.401,0.301)$ \\
Expert $_{2}$ & $(0.721,0.244)$ & $(0.381,0.624)$ & $(0.574,0.567)$ & $(0.213,0.733)$ \\
Expert $_{3}$ & $(0.520,0.330)$ & $(0.460,0.770)$ & $(0.670,0.540)$ & $(0.440,0.620)$ \\
Expert $_{4}$ & $(0.780,0.372)$ & $(0.332,0.672)$ & $(0.822,0.472)$ & $(0.722,0.132)$ \\
Expert $_{5}$ & $(0.882,0.121)$ & $(0.881,0.214)$ & $(0.784,0.234)$ & $(0.652,0.652)$ \\
\hline
\end{tabular}

Table 9 Table showing explanations of software $\Delta_{7}$

\begin{tabular}{|c|c|c|c|c|}
\hline & IP & $\mathrm{ME}$ & DEM/DSM & $\mathrm{PM} / \mathrm{TA}$ \\
\hline Expert $_{1}$ & $(0.621,0.261)$ & $(0.571,0.292)$ & $(0.522,0.362)$ & $(0.722,0.131)$ \\
\hline Expert $_{2}$ & $(0.651,0.251)$ & $(0.732,0.362)$ & $(0.772,0.361)$ & $(0.731,0.231)$ \\
\hline Expert $_{3}$ & $(0.681,0.162)$ & $(0.892,0.272)$ & $(0.661,0.321)$ & $(0.751,0.142)$ \\
\hline Expert $_{4}$ & $(0.670,0.240)$ & $(0.860,0.321)$ & $(0.780,0.330)$ & $(0.660,0.130)$ \\
\hline Expert $_{5}$ & $(0.520,0.120)$ & $(0.610,0.240)$ & $(0.640,0.460)$ & $(0.870,0.230)$ \\
\hline
\end{tabular}

Table 10 Table showing explanations of software $\Delta_{8}$

\begin{tabular}{|lllll} 
& IP & ME & DEM/DSM & PM/TA \\
& & & & \\
Expert $_{1}$ & $(0.241,0.481)$ & $(0.641,0.352)$ & $(0.561,0.122)$ & $(0.591,0.311)$ \\
Expert $_{2}$ & $(0.881,0.242)$ & $(0.262,0.573)$ & $(0.567,0.611)$ & $(0.212,0.661)$ \\
Expert $_{3}$ & $(0.871,0.132)$ & $(0.342,0.783)$ & $(0.573,0.573)$ & $(0.442,0.722)$ \\
Expert $_{4}$ & $(0.661,0.471)$ & $(0.232,0.712)$ & $(0.342,0.621)$ & $(0.321,0.711)$ \\
Expert $_{5}$ & $(0.552,0.732)$ & $(0.561,0.461)$ & $(0.531,0.622)$ & $(0.212,0.782)$ \\
\hline
\end{tabular}


Table 11 Table showing explanations of software $\Delta_{9}$

$\begin{array}{lllll} & \text { IP } & \text { ME } & \text { DEM/DSM } & \text { PM/TA } \\ & & & & \\ \text { Expert }_{1} & (0.574,0.174) & (0.554,0.060) & (0.550,0.190) & (0.420,0.420) \\ \text { Expert }_{2} & (0.821,0.121) & (0.212,0.782) & (0.661,0.231) & (0.232,0.872) \\ \text { Expert }_{3} & (0.820,0.001) & (0.770,0.120) & (0.770,0.140) & (0.890,0.230) \\ \text { Expert }_{4} & (0.780,0.330) & (0.330,0.780) & (0.540,0.720) & (0.110,0.780) \\ \text { Expert }_{5} & (0.131,0.671) & (0.330,0.780) & (0.560,0.680) & (0.321,0.771)\end{array}$

Table 12 Table showing explanations of software $\Delta_{10}$

\begin{tabular}{|c|c|c|c|c|}
\hline & IP & ME & DEM/DSM & $\mathrm{PM} / \mathrm{TA}$ \\
\hline Expert $_{1}$ & $(0.45,0.23)$ & $(0.51,0.11)$ & $(0.56,0.26)$ & $(0.88,0.13)$ \\
\hline Expert $_{2}$ & $(0.83,0.12)$ & $(0.33,0.82)$ & $(0.76,0.32)$ & $(0.45,0.87)$ \\
\hline Expert $_{3}$ & $(0.89,0.23)$ & $(0.73,0.34)$ & $(0.89,0.30)$ & $(0.17,0.82)$ \\
\hline Expert $_{4}$ & $(0.56,0.17)$ & $(0.78,0.12)$ & $(0.88,0.42)$ & $(0.77,0.12)$ \\
\hline Expert $_{5}$ & $(0.47,0.66)$ & $(0.67,0.21)$ & $(0.66,0.22)$ & $(0.78,0.11)$ \\
\hline
\end{tabular}

Step 1 From the tables 3, 4, , 12, PFSS representation of software $\Delta_{x}(x=1,2, \cdots 10)$ can be done easily where, universal set is $\{\mathrm{IP}, \mathrm{ME}, \mathrm{DEM} / \mathrm{DSM}, \mathrm{PM} / \mathrm{TA}\}$ and parameter set is $\left\{\right.$ Expert $_{1}$, Expert $_{2}$, Expert $_{3}$, Expert $_{4}$, Expert $\left._{5}\right\}$.

Step 2 The similarity matrix is framed using $\Psi_{3}\left(\Delta_{x}, \Delta_{y}\right)$.

$$
\mathcal{M}=\left(\begin{array}{lllllllllll}
1.0000 & 0.8362 & 0.8100 & 0.7850 & 0.7860 & 0.7210 & 0.7796 & 0.69657 & 0.6911 & 0.7423 \\
0.8362 & 1.0000 & 0.7792 & 0.7527 & 0.7741 & 0.7094 & 0.7537 & 0.6962 & 0.7070 & 0.7121 \\
0.8100 & 0.7792 & 1.0000 & 0.7593 & 0.7857 & 0.6947 & 0.7455 & 0.6986 & 0.7332 & 0.7201 \\
0.7850 & 0.7527 & 0.7593 & 1.0000 & 0.7430 & 0.6668 & 0.7723 & 0.6599 & 0.6744 & 0.7613 \\
0.7860 & 0.7741 & 0.7857 & 0.7430 & 1.0000 & 0.7113 & 0.7562 & 0.6423 & 0.6854 & 0.7033 \\
0.7210 & 0.7094 & 0.6947 & 0.6668 & 0.7113 & 1.0000 & 0.6968 & 0.7249 & 0.6632 & 0.6778 \\
0.7796 & 0.7537 & 0.7455 & 0.7723 & 0.7562 & 0.6968 & 1.0000 & 0.6275 & 0.6530 & 0.7269 \\
0.6965 & 0.6962 & 0.6986 & 0.6599 & 0.6423 & 0.7249 & 0.6275 & 1.0000 & 0.7474 & 0.6661 \\
0.6911 & 0.7070 & 0.7332 & 0.6744 & 0.6854 & 0.6632 & 0.6530 & 0.7474 & 1.0000 & 0.6787 \\
0.7423 & 0.7121 & 0.7201 & 0.7613 & 0.7033 & 0.6778 & 0.7269 & 0.6661 & 0.6787 & 1.0000
\end{array}\right)
$$

Step 3 Calculate $\mathcal{M}^{2}=\mathcal{M} \circ \mathcal{M}$

$$
\mathcal{M}^{2}=\left(\begin{array}{lllllllllll}
1.0000 & 0.8362 & 0.8100 & 0.7850 & 0.7860 & 0.7210 & 0.7796 & 0.7210 & 0.7332 & 0.7613 \\
0.8362 & 1.0000 & 0.8100 & 0.7850 & 0.7860 & 0.7210 & 0.7796 & 0.7094 & 0.7332 & 0.7527 \\
0.8100 & 0.8100 & 1.0000 & 0.7850 & 0.7860 & 0.7210 & 0.7796 & 0.7332 & 0.7332 & 0.7593 \\
0.7850 & 0.7850 & 0.7850 & 1.0000 & 0.7850 & 0.7210 & 0.7796 & 0.6986 & 0.7332 & 0.7613 \\
0.7860 & 0.7860 & 0.7860 & 0.7850 & 1.0000 & 0.7210 & 0.7796 & 0.7113 & 0.7332 & 0.7430 \\
0.7210 & 0.7210 & 0.7210 & 0.7210 & 0.7210 & 1.0000 & 0.7210 & 0.7249 & 0.7249 & 0.7210 \\
0.7796 & 0.7796 & 0.7796 & 0.7796 & 0.7796 & 0.7210 & 1.0000 & 0.6986 & 0.7332 & 0.7613 \\
0.7210 & 0.7094 & 0.7332 & 0.6986 & 0.7113 & 0.7249 & 0.6986 & 1.0000 & 0.7474 & 0.6986 \\
0.7332 & 0.7332 & 0.7332 & 0.7332 & 0.7332 & 0.7249 & 0.7332 & 0.7474 & 1.0000 & 0.7201 \\
0.7613 & 0.7527 & 0.7593 & 0.7613 & 0.7430 & 0.7210 & 0.7613 & 0.6986 & 0.7201 & 1.0000
\end{array}\right)
$$


Calculate $\mathcal{M}^{4}=\mathcal{M}^{2} \circ \mathcal{M}^{2}$

$$
\mathcal{M}^{4}=\left(\begin{array}{lllllllllll}
1.0000 & 0.8362 & 0.8100 & 0.7850 & 0.7860 & 0.7249 & 0.7796 & 0.7332 & 0.7332 & 0.7613 \\
0.8362 & 1.0000 & 0.8100 & 0.7850 & 0.7860 & 0.7249 & 0.7796 & 0.7332 & 0.7332 & 0.7613 \\
0.8100 & 0.8100 & 1.0000 & 0.7850 & 0.7860 & 0.7249 & 0.7796 & 0.7332 & 0.7332 & 0.7613 \\
0.7850 & 0.7850 & 0.7850 & 1.0000 & 0.7850 & 0.7249 & 0.7796 & 0.7332 & 0.7332 & 0.7613 \\
0.7860 & 0.7860 & 0.7860 & 0.7850 & 1.0000 & 0.7249 & 0.7796 & 0.7332 & 0.7332 & 0.7613 \\
0.7249 & 0.7249 & 0.7249 & 0.7249 & 0.7249 & 1.0000 & 0.7249 & 0.7249 & 0.7249 & 0.7210 \\
0.7796 & 0.7796 & 0.7796 & 0.7796 & 0.7796 & 0.7249 & 1.0000 & 0.7332 & 0.7332 & 0.7613 \\
0.7332 & 0.7332 & 0.7332 & 0.7332 & 0.7332 & 0.7249 & 0.7332 & 1.0000 & 0.7474 & 0.7332 \\
0.7332 & 0.7332 & 0.7332 & 0.7332 & 0.7332 & 0.7249 & 0.7332 & 0.7474 & 1.0000 & 0.7332 \\
0.7613 & 0.7613 & 0.7613 & 0.7613 & 0.7613 & 0.7210 & 0.7613 & 0.7332 & 0.7332 & 1.0000
\end{array}\right)
$$

Calculate $\mathcal{M}^{8}=\mathcal{M}^{4} \circ \mathcal{M}^{4}$

$$
\mathcal{M}^{8}=\left(\begin{array}{lllllllllll}
1.0000 & 0.8362 & 0.8100 & 0.7850 & 0.7860 & 0.7249 & 0.7796 & 0.7332 & 0.7332 & 0.7613 \\
0.8362 & 1.0000 & 0.8100 & 0.7850 & 0.7860 & 0.7249 & 0.7796 & 0.7332 & 0.7332 & 0.7613 \\
0.8100 & 0.8100 & 1.0000 & 0.7850 & 0.7860 & 0.7249 & 0.7796 & 0.7332 & 0.7332 & 0.7613 \\
0.7850 & 0.7850 & 0.7850 & 1.0000 & 0.7850 & 0.7249 & 0.7796 & 0.7332 & 0.7332 & 0.7613 \\
0.7860 & 0.7860 & 0.7860 & 0.7850 & 1.0000 & 0.7249 & 0.7796 & 0.7332 & 0.7332 & 0.7613 \\
0.7249 & 0.7249 & 0.7249 & 0.7249 & 0.7249 & 1.0000 & 0.7249 & 0.7249 & 0.7249 & 0.7249 \\
0.7796 & 0.7796 & 0.7796 & 0.7796 & 0.7796 & 0.7249 & 1.0000 & 0.7332 & 0.7332 & 0.7613 \\
0.7332 & 0.7332 & 0.7332 & 0.7332 & 0.7332 & 0.7249 & 0.7332 & 1.0000 & 0.7474 & 0.7332 \\
0.7332 & 0.7332 & 0.7332 & 0.7332 & 0.7332 & 0.7249 & 0.7332 & 0.7474 & 1.0000 & 0.7332 \\
0.7613 & 0.7613 & 0.7613 & 0.7613 & 0.7613 & 0.7249 & 0.7613 & 0.7332 & 0.7332 & 1.0000
\end{array}\right)
$$

Calculate $\mathcal{M}^{16}=\mathcal{M}^{8} \circ \mathcal{M}^{8}$

$$
\mathcal{M}^{16}=\left(\begin{array}{lllllllllll}
1.0000 & 0.8362 & 0.8100 & 0.7850 & 0.7860 & 0.7249 & 0.7796 & 0.7332 & 0.7332 & 0.7613 \\
0.8362 & 1.0000 & 0.8100 & 0.7850 & 0.7860 & 0.7249 & 0.7796 & 0.7332 & 0.7332 & 0.7613 \\
0.8100 & 0.8100 & 1.0000 & 0.7850 & 0.7860 & 0.7249 & 0.7796 & 0.7332 & 0.7332 & 0.7613 \\
0.7850 & 0.7850 & 0.7850 & 1.0000 & 0.7850 & 0.7249 & 0.7796 & 0.7332 & 0.7332 & 0.7613 \\
0.7860 & 0.7860 & 0.7860 & 0.7850 & 1.0000 & 0.7249 & 0.7796 & 0.7332 & 0.7332 & 0.7613 \\
0.7249 & 0.7249 & 0.7249 & 0.7249 & 0.7249 & 1.0000 & 0.7249 & 0.7249 & 0.7249 & 0.7249 \\
0.7796 & 0.7796 & 0.7796 & 0.7796 & 0.7796 & 0.7249 & 1.0000 & 0.7332 & 0.7332 & 0.7613 \\
0.7332 & 0.7332 & 0.7332 & 0.7332 & 0.7332 & 0.7249 & 0.7332 & 1.0000 & 0.7474 & 0.7332 \\
0.7332 & 0.7332 & 0.7332 & 0.7332 & 0.7332 & 0.7249 & 0.7332 & 0.7474 & 1.0000 & 0.7332 \\
0.7613 & 0.7613 & 0.7613 & 0.7613 & 0.7613 & 0.7249 & 0.7613 & 0.7332 & 0.7332 & 1.0000
\end{array}\right)
$$

It is obtained that $\mathcal{M}^{8}=\mathcal{M}^{16}$. Thus the equivalent similarity matrix is identified as $\mathcal{M}^{8}$.

Step 4 Let the confidence level $\Gamma=0.7860$. The $\Gamma$-cutting matrix $\mathcal{M}^{\Gamma}$ is obtained as,

$$
\mathcal{M}^{\Gamma}=\left(\begin{array}{llllllllll}
1 & 1 & 1 & 0 & 1 & 0 & 0 & 0 & 0 & 0 \\
1 & 1 & 1 & 0 & 1 & 0 & 0 & 0 & 0 & 0 \\
1 & 1 & 1 & 0 & 1 & 0 & 0 & 0 & 0 & 0 \\
0 & 0 & 0 & 1 & 0 & 0 & 0 & 0 & 0 & 0 \\
1 & 1 & 1 & 0 & 1 & 0 & 0 & 0 & 0 & 0 \\
0 & 0 & 0 & 0 & 0 & 1 & 0 & 0 & 0 & 0 \\
0 & 0 & 0 & 0 & 0 & 0 & 1 & 0 & 0 & 0 \\
0 & 0 & 0 & 0 & 0 & 0 & 0 & 1 & 0 & 0 \\
0 & 0 & 0 & 0 & 0 & 0 & 0 & 0 & 1 & 0 \\
0 & 0 & 0 & 0 & 0 & 0 & 0 & 0 & 0 & 1
\end{array}\right)
$$


Step 5 The classification of $\Delta_{x}(x=1,2, \cdots 10)$ s by using $\mathcal{M}^{\Gamma}$ matrix is obtained as, $\left\{\Delta_{1}, \Delta_{2}, \Delta_{3}, \Delta_{5}\right\},\left\{\Delta_{4}\right\},\left\{\Delta_{6}\right\},\left\{\Delta_{7}\right\},\left\{\Delta_{8}\right\},\left\{\Delta_{9}\right\},\left\{\Delta_{10}\right\}$.

Thus, under the given criteria with confidence level $\Gamma=0.7860$, we are able to conclude that the data processing and analysis of software $\Delta_{1}, \Delta_{2}, \Delta_{3}$ and $\Delta_{5}$ come under the same group, and each of the remaining ones form single groups.

Table 13 Table showing clustering of $\Delta_{x} \mathrm{~s}$ for different confidence level

\begin{tabular}{|c|c|c|}
\hline Confidence level & Clusters & $\begin{array}{l}\text { Number of } \\
\text { Classes }\end{array}$ \\
\hline $0.0000 \leq \Gamma \leq 0.7249$ & $\left\{\Delta_{1}, \Delta_{2}, \Delta_{3}, \Delta_{4}, \Delta_{5}, \Delta_{6}, \Delta_{7}, \Delta_{8}, \Delta_{9}, \Delta_{10}\right\}$ & 1 \\
\hline $0.7249<\Gamma<0.7332$ & $\left\{\Delta_{1}, \Delta_{2}, \Delta_{3}, \Delta_{4}, \Delta_{5}, \Delta_{7}, \Delta_{8}, \Delta_{9}, \Delta_{10}\right\},\left\{\Delta_{6}\right\}$ & 2 \\
\hline $0.7332<\Gamma \overline{\leq} 0.7613$ & $\left\{\Delta_{1}, \Delta_{2}, \Delta_{3}, \Delta_{4}, \Delta_{5}, \Delta_{7}, \Delta_{10}\right\},\left\{\Delta_{6}\right\},\left\{\Delta_{8}, \Delta_{9}\right\}$ & 3 \\
\hline $0.7613<\Gamma \leq 0.7796$ & $\left\{\Delta_{1}, \Delta_{2}, \Delta_{3}, \Delta_{4}, \Delta_{5}, \Delta_{7}\right\},\left\{\Delta_{6}\right\},\left\{\Delta_{8}\right\},\left\{\Delta_{9}\right\},\left\{\Delta_{10}\right\}$ & 5 \\
\hline $0.7796<\Gamma \leq 0.7850$ & $\left\{\Delta_{1}, \Delta_{2}, \Delta_{3}, \Delta_{4}, \Delta_{5}\right\},\left\{\Delta_{6}\right\},\left\{\Delta_{7}\right\},\left\{\Delta_{8}\right\},\left\{\Delta_{9}\right\},\left\{\Delta_{10}\right\}$ & 6 \\
\hline $0.7850<\Gamma \leq 0.7860$ & $\left\{\Delta_{1}, \Delta_{2}, \Delta_{3}, \Delta_{5}\right\},\left\{\Delta_{4}\right\},\left\{\Delta_{6}\right\},\left\{\Delta_{7}\right\},\left\{\Delta_{8}\right\},\left\{\Delta_{9}\right\},\left\{\Delta_{10}\right\}$ & 7 \\
\hline $0.7860<\Gamma \leq 0.8100$ & $\left\{\Delta_{1}, \Delta_{2}, \Delta_{3}\right\},\left\{\Delta_{4}\right\},\left\{\Delta_{5}\right\},\left\{\Delta_{6}\right\},\left\{\Delta_{7}\right\},\left\{\Delta_{8}\right\},\left\{\Delta_{9}\right\},\left\{\Delta_{10}\right\}$ & 8 \\
\hline $0.8100<\Gamma \leq 0.8362$ & $\left\{\Delta_{1}, \Delta_{2}\right\},\left\{\Delta_{3}\right\}\left\{\Delta_{4}\right\},\left\{\Delta_{5}\right\},\left\{\Delta_{6}\right\},\left\{\Delta_{7}\right\},\left\{\Delta_{8}\right\},\left\{\Delta_{9}\right\},\left\{\Delta_{10}\right\}$ & 9 \\
\hline $0.8362<\Gamma \leq 1.0000$ & $\left\{\Delta_{1}\right\},\left\{\Delta_{2}\right\},\left\{\Delta_{3}\right\},\left\{\Delta_{4}\right\},\left\{\Delta_{5}\right\},\left\{\Delta_{6}\right\},\left\{\Delta_{7}\right\},\left\{\Delta_{8}\right\},\left\{\Delta_{9}\right\},\left\{\Delta_{10}\right\}$ & 10 \\
\hline
\end{tabular}

Since the $\mathcal{M}^{\Gamma}$ matrix varies according to different confidence levels, we obtain different clustering results. From Table 13, it is obtained that as the confidence level increases, the number of patterns obtained also increases. The clustering effect diagram (Figure 1) obtained by analysing the table 13 and we can conclude that software are separated mainly into the following two directions, $\left\{\Delta_{1}, \Delta_{2}, \Delta_{3}, \Delta_{4}, \Delta_{5}, \Delta_{7}, \Delta_{8}, \Delta_{9}, \Delta_{10}\right\}$ and $\left\{\Delta_{6}\right\}$. Thus, one can use the clustering effect diagram to take decisions in the above problem with a prescribed confidence level. Since in the proposed clustering algorithm we considered the opinions of five experts for choosing the software, it will be more accurate than the present algorithms. 
$\begin{array}{llllllllll}\Delta_{1} & \Delta_{3} & \Delta_{2} & \Delta_{4} & \Delta_{5} & \Delta_{7} & \Delta_{6} & \Delta_{10} & \Delta_{8} & \Delta_{9}\end{array}$

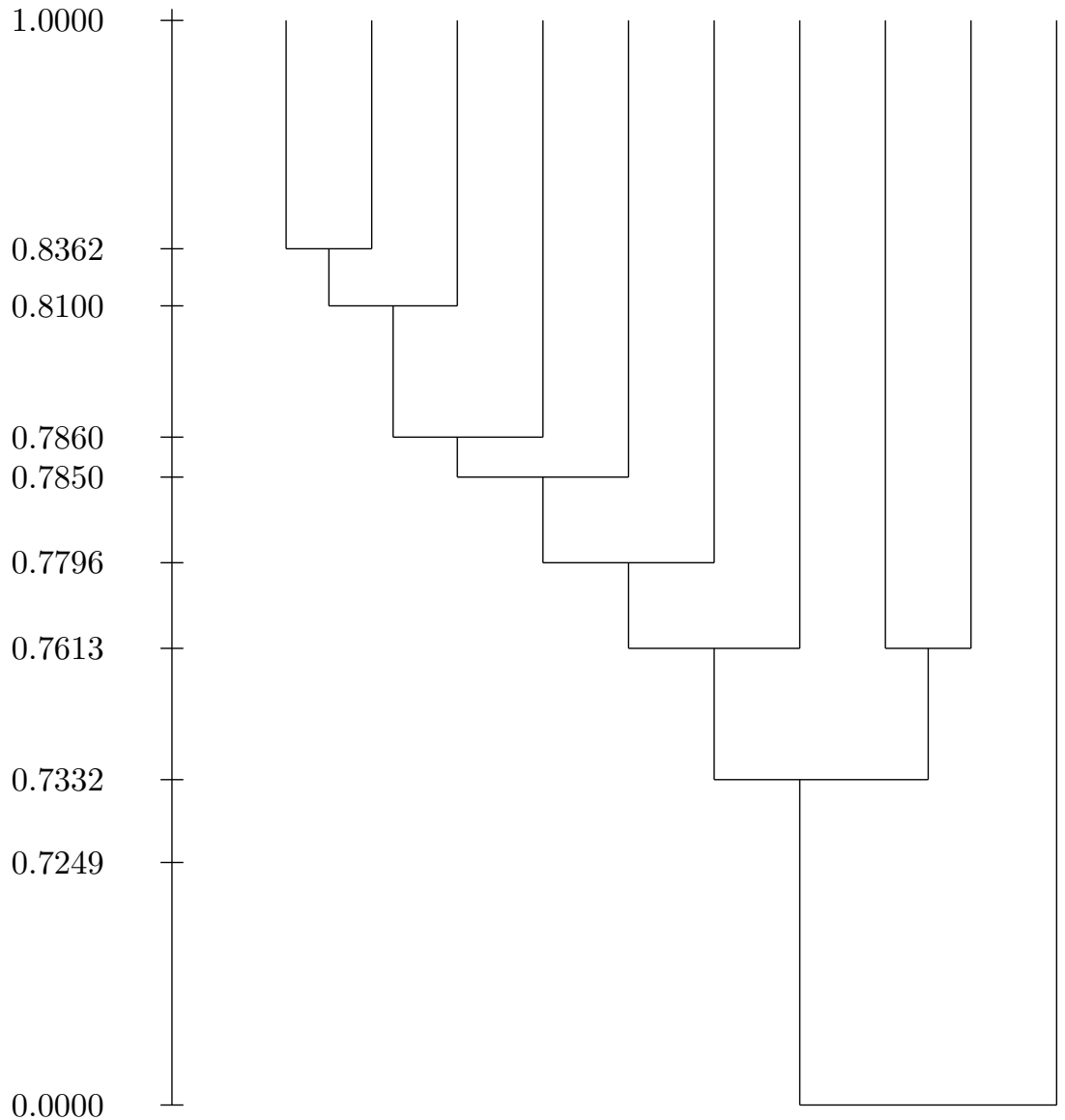

Fig.1 Clustering effect diagram corresponding to Ten software

\section{Conclusion}

This paper put forward the notion of similarity measure for PFSSs. Different expressions to compute similarity measures are obtained and a comparative study is executed. Finally, a real-life application, viz. cluster analysis is explained. This paper can fill up the deficiency of a measuring tool for finding similarities of objects when the vagueness is represented as PFSSs. We plan to study the concept of information measures of PFSS which are useful in decision-making problems in future. 


\section{Acknowledgment}

The first author would like to thank MHRD, Government of India along with National Institute of Technology, Calicut for the financial assistance and the DST, Government of India, for providing support to carry out this work under the scheme 'FIST' (No. SR/FST/MS-I/2019/40). The authors want to thank the editor and reviewers for their valuable and constructive comments to improve this paper.

\section{Declarations}

Funding This research received no external funding.

Conflicts of interest/Competing interests The authors declare that they have no conflict of interest.

Availability of data and material Not applicable

Code availability Not applicable

Authors' contributions ATM wrote the original manuscript. SJJ and HG contributed to writing, reviewing, and editing.

Ethical approval This article does not contain any studies with human participants or animals performed by any of the authors.

Consent to participate Informed consent was obtained from all individual participants included in the study.

Consent for publication Not applicable.

\section{Data availability statement}

Data sharing not applicable to this article as no data sets were generated or analysed during the current study.

\section{References}

1. Aktaş, H., Çă̆man, N.: Soft sets and soft groups. Information sciences 177(13), 2726$2735(2007)$

2. Atanassov, K.T.: Intuitionistic fuzzy sets. Fuzzy sets and Systems 20(1), 87-96 (1986)

3. Athira, T., John, S.J., Kumar, P.R.: Incomplete pythagorean fuzzy soft sets. In: AIP Conference Proceedings, vol. 2261, p. 030143. AIP Publishing LLC (2020)

4. Athira, T.M., John, S.J., Garg, H.: Entropy and distance measures of pythagorean fuzzy soft sets and their applications. Journal of Intelligent \& Fuzzy Systems (Preprint), 1-14 (2019)

5. Athira, T.M., John, S.J., Garg, H.: A novel entropy measure of pythagorean fuzzy soft sets. AIMS Mathematics 5(2), 1050-1061 (2019)

6. Babitha, K., Sunil, J.: Soft set relations and functions. Computers \& Mathematics with Applications 60(7), 1840-1849 (2010)

7. Că̆man, N., Deli, I.: Similarity measures of intuitionistic fuzzy soft sets and their decision making. arXiv preprint arXiv:1301.0456 (2013)

8. Cagman, N., Enginoglu, S., Citak, F.: Fuzzy soft set theory and its applications. Iranian Journal of Fuzzy Systems 8(3), 137-147 (2011) 
9. Garg, H.: A new generalized pythagorean fuzzy information aggregation using einstein operations and its application to decision making. International Journal of Intelligent Systems 31(9), 886-920 (2016)

10. Garg, H.: Confidence levels based pythagorean fuzzy aggregation operators and its application to decision-making process. Computational and Mathematical Organization Theory 23(4), 546-571 (2017)

11. Garg, H., Rani, D.: Some results on information measures for complex intuitionistic fuzzy sets. International Journal of Intelligent Systems 34(10), 2319-2363 (2019)

12. Guleria, A., Bajaj, R.K.: On pythagorean fuzzy soft matrices, operations and their applications in decision making and medical diagnosis. Soft Computing 23(17), 78897900 (2019)

13. Hameed, M.S., Mukhtar, S., Khan, H.N., Ali, S., Mateen, M.H., Gulzar, M.: Pythagorean fuzzy n-soft groups. International Journal of Electrical and Computer Engineering (IJECE) 21(2), 1030-1038 (2021)

14. Hong, D.H., Hwang, S.Y.: A note on the value similarity of fuzzy systems variables. Fuzzy Sets and Systems 66(3), 383-386 (1994)

15. John, S.J.: Soft sets. In: Soft Sets, pp. 3-36. Springer (2021)

16. Kharal, A.: Distance and similarity measures for soft sets. New Mathematics and Natural Computation 6(03), 321-334 (2010)

17. Liang, Z., Shi, P.: Similarity measures on intuitionistic fuzzy sets. Pattern Recognition Letters 24(15), 2687-2693 (2003)

18. Maji, P., Biswas, R., Roy, A.: Soft set theory. Computers \& Mathematics with Applications 45(4-5), 555-562 (2003)

19. Maji, P.K., Biswas, R., Roy, A.R.: Intuitionistic fuzzy soft sets. Journal of fuzzy mathematics 9(3), 677-692 (2001)

20. Majumdar, P., Samanta, S.: On similarity measures of fuzzy soft sets. International Journal of Advance Soft Computing and Applications 3(2), 1-8 (2011)

21. Majumdar, P., Samanta, S.K.: Similarity measure of soft sets. New mathematics and natural computation 4(01), 1-12 (2008)

22. Molodtsov, D.: Soft set theory - first results. Computers \& Mathematics with Applications 37(4-5), 19-31 (1999)

23. Mukherjee, A., Sarkar, S.: Distance based similarity measures for interval-valued intuitionistic fuzzy soft sets and its application. New Trends in Mathematical Sciences 3(4), 34-42 (2015)

24. Muthukumar, P., Krishnan, G.S.S.: A similarity measure of intuitionistic fuzzy soft sets and its application in medical diagnosis. Applied Soft Computing 41, 148-156 (2016)

25. Öztürk, T.Y., Yolcu, A.: Some structures on pythagorean fuzzy soft topological spaces. In: 3rd International Conference on Mathematical and Related Sciences: Current trends and developments proceedings book, p. 154 (2020)

26. Peng, X., Garg, H.: Multiparametric similarity measures on pythagorean fuzzy sets with applications to pattern recognition. Applied Intelligence pp. 1-39 (2019)

27. Peng, X., Selvachandran, G.: Pythagorean fuzzy set: state of the art and future directions. Artificial Intelligence Review pp. 1-55 (2017)

28. Peng, X., Yang, Y., Song, J., Jiang, Y.: Pythagoren fuzzy soft set and its application. Computer Engineering 41(7), 224-229 (2015)

29. Sarala, N., Suganya, B.: An application of similarity measure of intuitionistic fuzzy soft set based on distance in medical diagnosis. Int J Sci Res 5(3), 559-563 (2016)

30. Torra, V.: Hesitant fuzzy sets. International Journal of Intelligent Systems 25(6), 529$539(2010)$

31. Wang, P.: Theory of fuzzy sets and their applications. Shanghai Science and Technology Publishing House, Shanghai (1982)

32. Xu, Z., Chen, J., Wu, J.: Clustering algorithm for intuitionistic fuzzy sets. Information Sciences 178(19), 3775-3790 (2008)

33. Xuecheng, L.: Entropy, distance measure and similarity measure of fuzzy sets and their relations. Fuzzy sets and systems 52(3), 305-318 (1992)

34. Yager, R.R., Abbasov, A.M.: Pythagorean membership grades, complex numbers, and decision making. International Journal of Intelligent Systems 28(5), 436-452 (2013)

35. Zadeh, L.A.: Fuzzy sets. Information and control 8(3), 338-353 (1965) 
36. Zeng, W., Li, D., Yin, Q.: Distance and similarity measures of pythagorean fuzzy sets and their applications to multiple criteria group decision making. International Journal of Intelligent Systems 33(11), 2236-2254 (2018) 